Review

\title{
Terahertz Vibrations and Hydrogen-Bonded Networks in Crystals
}

\author{
Masae Takahashi \\ Graduate School of Agricultural Science, Tohoku University, Sendai 981-8555, Japan; \\ E-Mail: masae@ fris.tohoku.ac.jp; Tel.: +81-22-795-4271; Fax: +81-22-795-7810
}

Received: 14 January 2014; in revised form: 3 March 2014 / Accepted: 4 March 2014 /

Published: 31 March 2014

\begin{abstract}
The development of terahertz technology in the last few decades has made it possible to obtain a clear terahertz $(\mathrm{THz})$ spectrum. THz vibrations clearly show the formation of weak bonds in crystals. The simultaneous progress in the code of first-principles calculations treating noncovalent interactions has established the position of $\mathrm{THz}$ spectroscopy as a powerful tool for detecting the weak bonding in crystals. In this review, we are going to introduce, briefly, the contribution of weak bonds in the construction of molecular crystals first, and then, we will review $\mathrm{THz}$ spectroscopy as a powerful tool for detecting the formation of weak bonds and will show the significant contribution of advanced computational codes in treating noncovalent interactions. From the second section, following the Introduction, to the seventh section, before the conclusions, we describe: (1) the crystal packing forces, the hydrogen-bonded networks and their contribution to the construction of organic crystals; (2) the $\mathrm{THz}$ vibrations observed in hydrogen-bonded molecules; (3) the computational methods for analyzing the $\mathrm{THz}$ vibrations of hydrogen-bonded molecules; (4) the dispersion correction and anharmonicity incorporated into the first-principles calculations and their effect on the peak assignment of the $\mathrm{THz}$ spectrum (5) the temperature dependence; and (6) the polarization dependence of the THz spectrum.
\end{abstract}

Keywords: terahertz spectroscopy; first-principles calculations; weak hydrogen bond; dispersion correction 


\section{Introduction}

Terahertz (THz) vibrations in the frequency range of $30-200 \mathrm{~cm}^{-1}$ give direct evidence for the formation of weak bonds in crystals. The THz region of the electromagnetic spectrum lies between the microwave and infrared (Figure 1) regions. The contributions and efforts in the field of materials research and crystal engineering [1-7], particularly those joined with the hydrogen bonding interactions in the field of THz spectroscopy, have resulted in a wide range of implementations of this method in practice in spectroscopic imaging [8], environmental inspection and detection [9], public security [10,11], biomedical analysis [12,13] and wireless communications [14-17]. The fundamental scientific contribution and wide extent of the THz-spectroscopic method's application results from the fact that $\mathrm{THz}$ radiation is transmitted through most non-metallic and non-polar mediums, thus enabling one to detect packaging, corrugated cardboard, clothes, shoes and book bags, in order to probe for potentially dangerous materials, in combination with minimal human health risk. The method enables the identification of explosives, chemical and biological agents through their characteristic fingerprint in the $\mathrm{THz}$ spectrum That weak intermolecular interactions in crystals provide a spectroscopic profile with strongly individual characteristics in the $\mathrm{THz}$ region, i.e., the $\mathrm{THz}$ method is very sensitive to the weak intermolecular interactions in crystals, enables the unambiguous identification of materials. Its applicability, including for the purposes of pharmaceutical analysis, in identifying biologically active samples has been demonstrated recently. This review is intended to give a coherent survey of the weak bond in assembling molecular crystals, of $\mathrm{THz}$ spectroscopy as a powerful tool for detecting the formation of weak bonds in crystals and of the significant contribution of advanced computational codes to analyze the $\mathrm{THz}$ spectrum. We especially focus on the stretching vibration of the weak hydrogen bond observed in the THz spectrum.

Figure 1. Low-energy vibrational modes in the terahertz (THz) region.

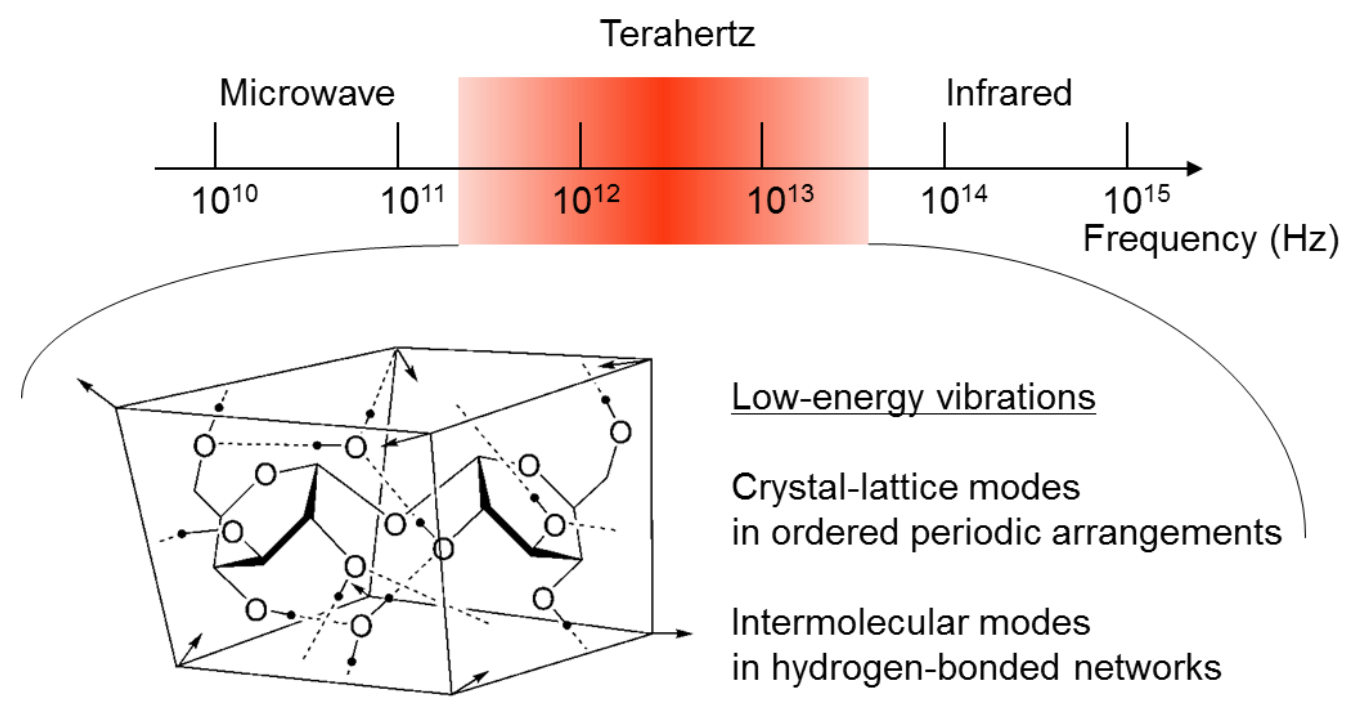

Due to the recent development of $\mathrm{THz}$ vibrational spectroscopy, the spectral range of the vibrational absorptions of organic molecules in the solid phase obtained with high resolution nowadays covers the crystal-lattice and intermolecular vibrations, in addition to the intramolecular fundamental vibrations (Figure 1). The applications of $\mathrm{THz}$ technology in probing the low-energy vibrational modes of 
materials have excited much interest [18-26]. Several recent breakthroughs made in light generation/detection have greatly contributed to the established $\mathrm{THz}$ spectroscopy [27-36]. Currently, the challenge and worldwide research activities around $\mathrm{THz}$ technologies have grown significantly with respect to the extension of the frequency range coverage and are related to interdisciplinary areas. The main efforts are focused on inorganic materials. Very recent findings in 2014 alone evidenced the perspectives of such studies [37-39]. Nevertheless, along with materials research and crystal engineering, i.e., the species interactions in crystals are focused mainly on inorganic materials, organic materials research also found a place in $\mathrm{THz}$ generation and detection. Such materials have shown great potential and broadband $\mathrm{THz}$ properties $\left(10-1000 \mathrm{~cm}^{-1}\right)$, extending the possibility of $\mathrm{THz}$ spectroscopy for the identification of materials in the mid-IR (infrared) region. Their application enables not only a qualitative fingerprinting analysis of materials, but also a quantitative one. Instrumental developments of $\mathrm{THz}$ spectrometers on the basis of organic materials have already been implemented. In this respect, the developments and contributions by Günter's group on nonlinear optical crystals have had a significant impact on the field of organic materials research and crystal engineering for applications in THz spectroscopy [40-46].

The analysis of the congested $\mathrm{THz}$ spectrum with vibrational absorption peaks appearing in the narrow wavenumber region of 30-200 $\mathrm{cm}^{-1}$, has been very challenging. The rapid development of computer power, together with the availability of efficient codes, has made the assignment of observed absorption peaks increasingly reliable [47,48]. Intermolecular interactions and global nuclear motions in the crystalline environment are now being revealed through the assigned $\mathrm{THz}$ vibrational mode. It has been pointed out that the stretching vibration of the weak hydrogen bond is located in the $\mathrm{THz}$ region [49]. Among intermolecular interactions, the weak hydrogen bond is very important for many structures and functions in chemical and biological systems [49-55].

Sections 2-8 consist of the following contents. Section 2 elaborates upon the packing force, which works in a crystal growth process and governs the crystal structure of organic molecules. The hydrogen bond among the packing forces is explained in detail, and polymorphs with different crystal packing patterns of the same molecules are introduced. Section 3 shows the $\mathrm{THz}$ vibrations of hydrogen-bonded systems observed experimentally. Section 4 states the minimum requirements of computational methods for analyzing the $\mathrm{THz}$ spectrum of hydrogen-bonded molecules. Section 5 describes additional corrections to the density functional theory (DFT) method, such as dispersive interaction and anharmonicity. Both are crucial in the analysis of the THz spectrum. Sections 6 and 7 introduce the temperature and polarization dependence of the $\mathrm{THz}$ spectrum. Conclusions are given in Section 8.

\section{Crystal Packing Forces and Hydrogen-Bonded Networks}

The packing forces to assemble molecular crystals in the solid state are noncovalent, such as hydrogen bonding and van der Waals (vdW) interactions (Figure 2). The "weak" intermolecular forces, like vdW or the hydrogen bond, play a significant role in a great variety of physical, chemical and biological phenomena. The effects of these forces manifest themselves, e.g., in the behavior of real gases, in the special properties of water or in the processes of protein folding. Consequently, a large number of the experimental and theoretical studies have been carried out in order to increase our 
knowledge about the nature of the intermolecular forces. It is interesting that different packing of the same molecules leads to the formation of a new polymorphic crystal structure.

Figure 2. Intermolecular interactions for determining the packing of organic molecules during the crystal-growth process.

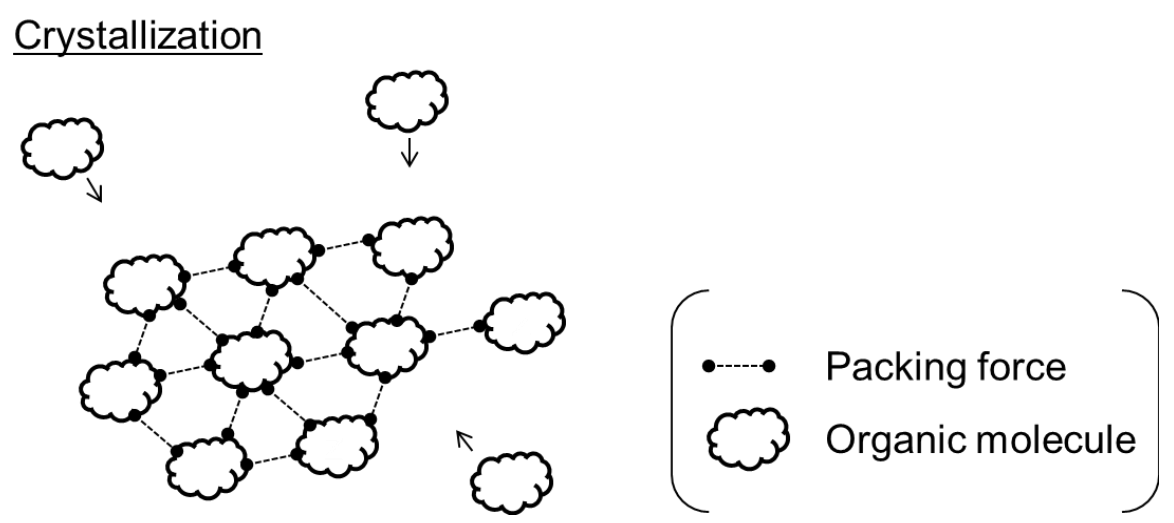

Hydrogen bonding was discovered almost 100 years ago [49-55], but still is a topic of vital scientific research. The reason for the long-standing interest lies in the eminent importance of hydrogen bonds for the structure, function and dynamics of a vast number of chemical systems, which range from inorganic to biological chemistry. The scientific branches involved are very diverse, and one may include mineralogy, materials science, general inorganic and organic chemistry, supermolecular chemistry, biochemistry, molecular medicine and pharmacy. In the hydrogen bond denoted by $\mathrm{X}-\mathrm{H} \cdots \mathrm{A}$, the group, $\mathrm{X}-\mathrm{H}$, is called proton donor or electron acceptor, and $\mathrm{A}$ is called the proton acceptor or electron donor. The chemical variation of the donor and/or acceptor, and possibly also of the environment, can gradually change the hydrogen bond to another interaction type. The transition to pure vdW interactions is very common. The hydrogen bond consists mainly of three interactions: electrostatic, induction and dispersion (Figure 3). The strength of electrostatic interactions depends on the polarity of $\mathrm{X}^{\delta^{-}}-\mathrm{H}^{\delta^{+}}$or $\mathrm{A}^{\delta^{-}}$. Induction is the interaction between a permanent dipole and an induced dipole. Dispersion is the interaction between induced dipoles. The polarity of $\mathrm{X}^{\delta_{-}-\mathrm{H}^{\delta+}}$ or $\mathrm{A}^{\delta^{-}}$(or both) in the array, $\mathrm{X}-\mathrm{H} \cdots \mathrm{A}$, can be reduced by the suitable variation of $\mathrm{X}$ or $\mathrm{A}$. This reduces the electrostatic part of the interaction, whereas the $\mathrm{vdW}$ component is much less affected. At the other end of the energy scale, there is a continuous transition to covalent bonding [56]. As a consequence, hydrogen bonds exist with a continuum of strengths. It can be useful for practical reasons to introduce a classification, such as "weak", "strong" and, possibly, also "in between". Jeffrey called hydrogen bonds moderate if they resemble those between water molecules or in carbohydrates (one could also call them "normal") and are associated with energies in the range of 4-15 $\mathrm{kcal} \cdot \mathrm{mol}^{-1}$ [57]. Hydrogen bonds with energies above and below this range are termed strong and weak, respectively. Weak and less common types of hydrogen bonds have been a major topic in hydrogen bond research. In the weak hydrogen bond of $\mathrm{X}-\mathrm{H} \cdots \mathrm{A}, \mathrm{X}$ and $\mathrm{A}$ are not simultaneously very electronegative atoms. The bond is very weak ( $c a .1 \mathrm{kcal} \cdot \mathrm{mol}^{-1}$ ), but it is important especially in the formation of molecular crystals or the higher order structure of proteins. The existence of the unconventional weak hydrogen bond was proposed early for the $\mathrm{C}-\mathrm{H} \cdots \mathrm{O}$ contact, where a hydrogen atom is covalently bonded to a less electronegative carbon atom [58]. After years of controversy, the final crystallographic evidence for 
the existence of $\mathrm{C}-\mathrm{H} \cdots \mathrm{A}(\mathrm{A}=\mathrm{O}, \mathrm{N}, \mathrm{Cl})$ hydrogen bonds was provided by a survey of 113 neutron diffraction structures [59].

Figure 3. Three kinds of interactions in hydrogen bonds.

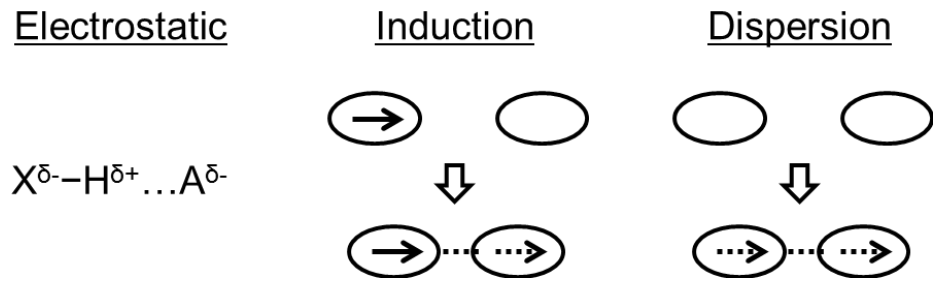

The THz spectra of crystalline solids are typically and uniquely sensitive to the molecular packing configurations, allowing for the detection of polymorphs. Polymorphism, the ability of solid material to exist in more than one form or crystal structure, is exceedingly important because of its relevance to structure-property relationships, the subtle and mysterious relationship between the structure and crystal packing of a molecule and the immense practical importance in the quality control of molecular materials industries, such as pharmaceuticals [60,61]. One is able to analyze and understand with increasing certainty a vast amount of accurate data currently available from crystallographic databases, while on the other hand, the appreciation of phenomena, like polymorphism, can lead to immediate benefits in applied areas, such as pharmaceutical development [62]. Many published works summarize the structural aspects of numerous polymorphic systems [63]. For distinguishing pharmaceuticals of a similar molecular composition, but differing crystal structures, $\mathrm{THz}$ spectroscopic and $\mathrm{THz}$ imaging studies on the polymorphism and/or cocrystal of several pharmaceuticals were intensively performed [64-72]. Here, spectral analysis with computational simulations is a key step for the distinction. A large number of molecules are found to exhibit polymorphism. The occurrence of polymorphic modifications in molecular compounds is manifested not only as a consequence of the minimum free energy in the crystalline phase, but also by the kinetics of the crystal nucleation and growth. Conformational polymorphs [73,74] are generated if the molecular conformation is different from one polymorph to the other; packing polymorphs [75] are those in which the molecules of a similar conformation pack into different crystal lattices and solvatomorphs (pseudopolymorphs) [76] that crystallize, including the solvents in the crystal lattice.

The polymorphs in which a weak hydrogen bond and/or other weak noncovalent interactions generate a distinct packing feature have been intensively investigated [77-87]. The second polymorph (Form II) of aspirin was recently found, and it is differentiated by the network of weak hydrogen bonds from the original form (Form I) [88]. The polymorphism of aspirin is, however, still an enigma. The molecular conformations are almost identical, showing only small differences of approximate two degrees in three dihedral angles, $C^{2}-C^{1}-C^{7}-O^{1}, C^{1}-C^{2}-O^{3}-C^{8}$ and $C^{2}-O^{3}-C^{8}-C^{9}$ (Figure 4). The crystal structure of aspirin Form I was first determined by Wheatley in 1964 [89], but the gas-phase structure of aspirin was unveiled very recently by the rotational study of supersonically cooled aspirin, using laser ablation molecular beam Fourier transform microwave spectroscopy and its conformational analysis [90]. Aspirin Form II was predicted in 2004 [91] and observed experimentally the following year. Form II is relatively stable at $100 \mathrm{~K}$, converting to the original Form I under room temperature. The crystal packing differs only slightly between the two polymorphs [92,93]. Aspirin 
Form I chains the carboxylic-acid and acetyl-group centrosymmetric dimers alternately in one dimension nearly along the $a$-axis (Figure 4a). An aspirin Form II dimer is connected via catemeric methyl $\mathrm{C}-\mathrm{H} \cdots \mathrm{O}$ (Figure 4b). It was later found that the Form II structure is completely superimposable on the Form I structure. A very recent computational study revealed that Forms I and II are virtually degenerate [94]. That is, the crystal structure of Forms I and II is indistinguishable in structure and energy after geometry optimization. The detection of the bonding that distinguishes two polymorphs is needed to settle the dispute, and the stretching vibration of the weak hydrogen bond can be detected in the THz region. The crystallization of aspirin with weak hydrogen-bonded networks is easily affected by additives. The growth-induced twisting of aspirin crystallites grown from the melt is driven by salicylic acid, either generated from aspirin decomposition or added deliberately [95]. Banded spherulites of twisted aspirin crystals grew as either of the two known polymorphs of aspirin or as a mixture of the two. The spherulites are composed of helicoidal crystallites twisted along the $<010>$ growth directions. The determination of the absolute sense of crystal growth in enantiopolar directions and the determination of the salicylic acid concentration variance in submicrometer-sized crystallites remain future experimental challenges.

Figure 4. The polymorphism of aspirin. (a) One-dimensional chain structure of aspirin Form I; and (b) the difference in the hydrogen-bonded networks of Forms I and II.

(a)

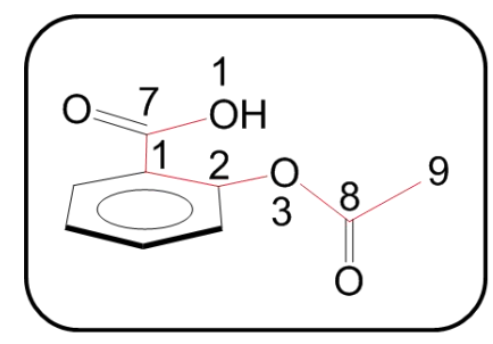

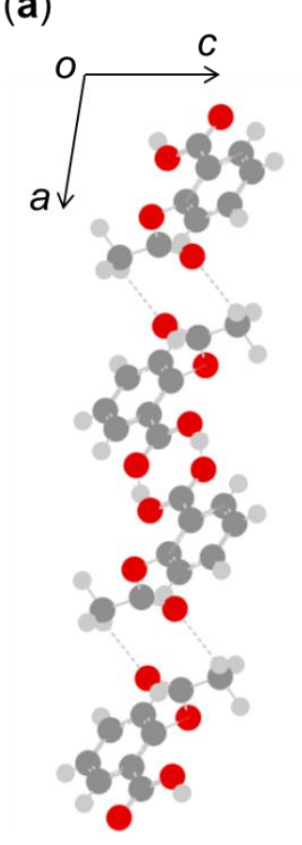

(b)

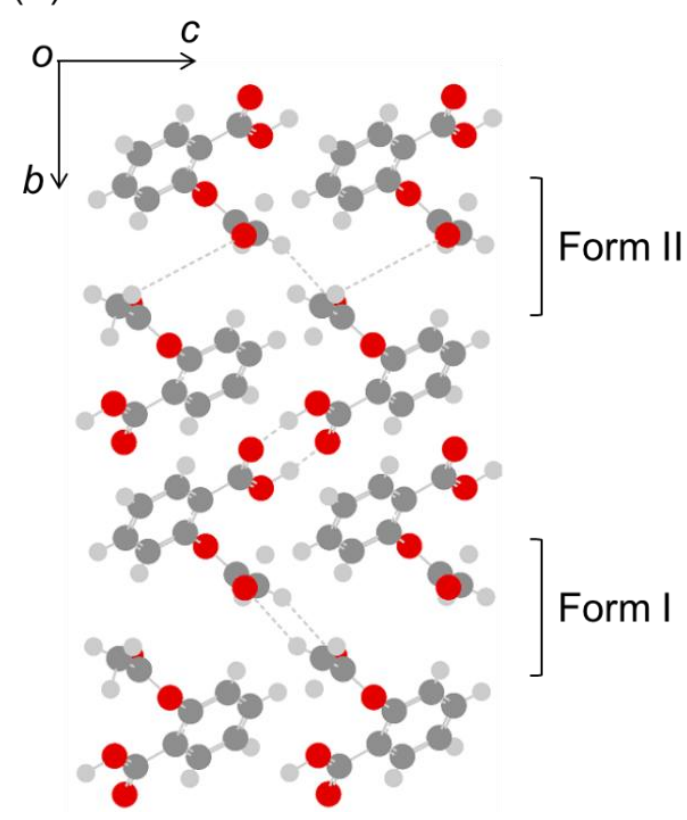

\section{THz Vibrations of Hydrogen-Bonded Molecules}

THz spectroscopy [31,36] is synonymous with far-infrared (FIR) spectroscopy, in the sense that both cover the low-frequency region below $200 \mathrm{~cm}^{-1}$. FIR spectroscopy first emerged in 1892 with the work of Rubens, but it took over seventy years to become widely available for scientific research [96,97]. The first commercial instruments were available in the 1960s, and extensive research in the vibrational spectroscopy of diverse organic molecules in the far- and mid-infrared frequencies proceeded over the next thirty years. Before 1990, there was a gap in the electromagnetic spectrum, 
3-50 $\mathrm{cm}^{-1}$, referred to as the "THz Gap", which was difficult to utilize, due to the lack of a suitable light source, and analysis using the frequencies between 50 and $500 \mathrm{~cm}^{-1}$ was usually referred to as FIR spectroscopy. The development of THz time-domain spectroscopy (THz-TDS) in the early 1990s was led by the initial discovery that $\mathrm{THz}$ pulses could be generated and detected $[98,99]$, and $\mathrm{THz}$ spectroscopy stimulated a lot of early interest. In 1993, tunable $\mathrm{THz}$ laser vibration-rotation-tunneling spectroscopy emerged as a powerful tool for quantifying the structure and hydrogen bond rearrangement dynamics of small water clusters at an unprecedented level of accuracy [100]. In 2005, a THz-TDS study was reported on a thin film of liquid $\mathrm{H}_{2} \mathrm{O}$, and a clear absorption mode was shown at $53 \mathrm{~cm}^{-1}$, attributed to the bending motion of intermolecular hydrogen bond coordinated in a water cage [101]. The advantages of $\mathrm{THz}$ spectroscopy for analytical purposes are its nondestructive nature, the use of nonionizing radiation, the short acquisition times and the simplicity of sample preparation. $\mathrm{The} \mathrm{THz}$ spectroscopy enables the characterization of solid-state materials through the excitation of soft intramolecular vibrational modes, as well as intermolecular modes and hydrogen-bonding networks inherent to the molecular assembly in the solid state [102,103].

The stretching vibration of the hydrogen bond in the $\mathrm{THz}$ region reflects the bonding itself [104-107]. The formation of a hydrogen bond affects the vibrational modes of the groups involved in several ways, and infrared (IR) spectroscopy is a standard method for investigating the hydrogen bond in the solid state [52]. In principle, H..A stretching vibration is the most direct spectroscopic indicator of hydrogen bonding. The frequency of stretching vibrations decreases as the interaction becomes weaker. The stretching vibration of a weaker kind of hydrogen bond appears in the THz region [49]. Reporting explicitly stating the stretching vibration of the weak hydrogen bond is very rare, despite the increase of publications on $\mathrm{THz}$ spectroscopic studies and their spectral analyses.

The $\mathrm{O}-\mathrm{H} \cdots \mathrm{O}$ stretching vibrations of water clusters are in the THz region. The X-ray study of water indicates the tetrahedral structure of the hydrogen-bonded water pentamer $[108,109]$. The entire structure of five-molecule water is described exactly by the $C_{2}$ point group (Figure 5). The water pentamer has 39 vibrational modes: 15 refer to intramolecular vibrations of $\mathrm{H}_{2} \mathrm{O}$, symmetric stretching and bending and asymmetric stretching fundamentals; 15 refer to three restricted rotations or librations of individual $\mathrm{H}_{2} \mathrm{O}$ molecules; and the remaining nine refer to hydrogen-bond stretching and bending. Each vibrational mode may be mixed, but the description is given by the main motion. The librational modes of water were observed at $450-780 \mathrm{~cm}^{-1}$ [110-112] by FIR and Raman spectroscopy. The O-H $\cdots$ O stretching vibrations were observed at 167 (FIR) [110] and 175 (Raman) $\mathrm{cm}^{-1}$ [112] and the $\mathrm{O}-\mathrm{H} \cdots \mathrm{O}$ bending vibrations at 53 (THz-TDS) [101] and 60 (Raman) $\mathrm{cm}^{-1}$ [112]. The IR spectrum of the water pentamer with $C_{2}$ symmetry calculated at Becke's three parameter exchange and Lee-Yang-Parr correlation functional (B3LYP) level using the correlation consistent polarized valence triple-zeta (cc-pVTZ) basis set [113,114] gives two strong peaks (Figure 5). From the displacement vectors, the peak at the lower frequency $\left(32 \mathrm{~cm}^{-1}\right)$ among the two is assigned to the $\mathrm{O}-\mathrm{H} \cdots \mathrm{O}$ bending vibration and the other peak at a higher frequency $\left(174 \mathrm{~cm}^{-1}\right)$ to the $\mathrm{O}-\mathrm{H} \cdots \mathrm{O}$ stretching vibration. 
Figure 5. Hydrogen-bonded water pentamer with $C_{2}$ symmetry (insert) and the infrared (IR) spectrum calculated at Becke's three parameter exchange and Lee-Yang-Parr correlation functional (B3LYP)/the correlation consistent polarized valence triple-zeta (cc-pVTZ) level.

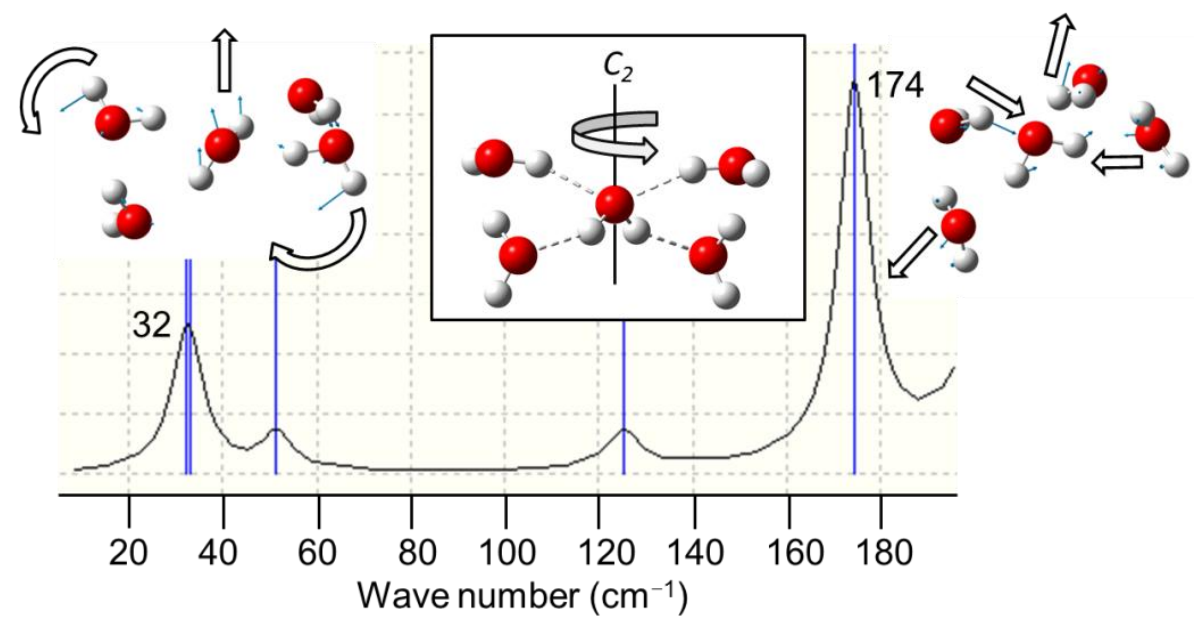

The stretching vibration of intermolecular hydrogen bonds appears as intermolecular translational modes and provides evidence for the bonding, but the translational vibrations in crystals themselves are observed in the spectrum irrespective of the existence of a hydrogen bond. The translational vibration mixed with the stretching mode of intermolecular hydrogen bonds would, however, be higher in frequency than unmixed translational vibration. Both types of translational vibrations between one-dimensional chains, mixed and unmixed with the stretching mode of the weak hydrogen bond between chains, are expected in aspirin Form I in the THz spectral region [72]. Aspirin has four molecules in the unit cell, and the one-dimensional chain is constructed nearly in the direction of the $a$-axis (Figure 4a). In addition to some intramonomer modes, twelve intermonomer modes, six interdimer (or inter-chain) modes and three lattice modes exist in the THz region. Two translational vibrations nearly along the $b$ - and $c$-axes are IR-active in the THz region (Figure 6). The relatively weak peak at $38 \mathrm{~cm}^{-1}$ in the low-temperature $\mathrm{THz}$ spectrum is assigned to the translational vibration nearly along the $b$-axis. One of the three strong peaks between 62 and $78 \mathrm{~cm}^{-1}$ is assigned to the translational vibration nearly along the $c$-axis. The translational vibration along the $b$-axis in the present unit cell does not contain the stretching vibration of the weak hydrogen bond between chains, where the one-dimensional chain shifts mutually in the direction of the $b$-axis, as indicated by the arrows in Figure 6. The translational vibration along the $c$-axis contains the approaching/receding motion of methyl hydrogen to/from the oxygen atom of the adjacent chain. The distance between the hydrogen and oxygen atoms (2.690 $\mathrm{\AA})$ [92] from X-ray crystallographic data is nearly equal to a classical vdW separation $(\sim 2.7 \AA)$ [115], supporting the existence of a weak hydrogen bond. The relatively strong peak assigned to the translational vibration in the direction of the $c$-axis is a candidate for the translation containing the stretching of the weak hydrogen bond. The frequency is higher than that of the other translational vibration containing no weak hydrogen bond stretching mode.

The inter-chain translational vibration mixed with the stretching of the hydrogen bond between the chains of poly-(R)-3-hydroxybutyrate (PHB) was observed near the translation of aspirin Form I containing the stretching of a weak hydrogen bond. PHB takes a lamellar helical structure in its crystal 
form [116,117]. The band at $\sim 80 \mathrm{~cm}^{-1}$ in the temperature- and polarization-dependent $\mathrm{THz}$ spectra was perpendicularly polarized to the helical axis, suggesting its origin in intermolecular hydrogen bonds among the PHB chains [118]. On the other hand, weak peaks were observed around $40 \mathrm{~cm}^{-1}$ in the low-temperature $\mathrm{THz}$ spectrum of benzoic acid, and those are assigned to inter-layer translational modes unmixed with the stretching vibration of the hydrogen bond [119]. The frequency is near the unmixed inter-chain translational vibration of aspirin. Benzoic acid has a hydrogen atom instead of the acetoxy group of aspirin at the ortho position. The crystal has four molecules in the unit cell, and two of four form a centrosymmetric dimer. The dimers in the crystal are connected by weak hydrogen bonds and construct a layered structure in two dimensions. Two dimers in the unit cell are, however, not in the same layer, and no hydrogen bond is constructed between the dimers in different layers. The interdimer translational vibration, which is an inter-layer translation, contains no stretching vibration of a weak hydrogen bond. It turns out that the translational vibration mixed with the stretching vibration of the hydrogen bond, as in $\mathrm{PHB}$, is higher in frequency than the unmixed translational vibrations, as in benzoic acid.

Figure 6. The $\mathrm{THz}$ spectrum of aspirin at $4 \mathrm{~K}$, and the displacement vector of two modes assigned to translational vibrations [72]. The arrows indicate the displacement of chains nearly along the $b$-axis (left) and the $c$-axis (right).

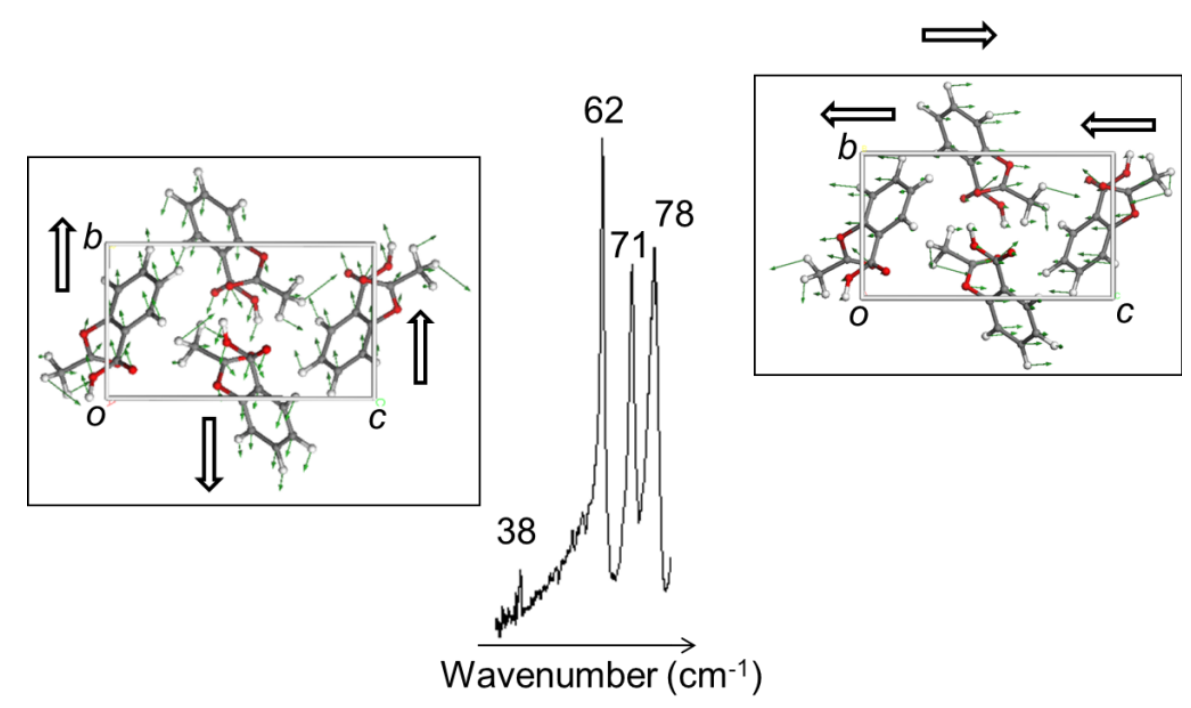

Low-frequency vibrational modes of protic ionic liquids (PIL) and ionic liquids (ILs) detect and quantify hydrogen bonds [120]. PILs are a subset of ILs and are formed by the combination of equimolar amounts of a Brønsted acid and a Brønsted base [121-128]. Hydrogen bonding in PILs is reminiscent of water [126]. The FIR spectra of dried PILs (ethylammonium nitrate, propylammonium nitrate and dimethylammonium nitrate) at $353 \mathrm{~K}$ give the asymmetric and symmetric stretching modes of the hydrogen bonds $\mathrm{N}-\mathrm{H} \cdots \mathrm{O}$ around $199-224 \mathrm{~cm}^{-1}$ and around $134-159 \mathrm{~cm}^{-1}$ and the bending modes of the hydrogen bonds around $60-78 \mathrm{~cm}^{-1}$. The measurement of the complex dielectric function of the PIL ethylammonium nitrate reveals a $\mathrm{THz}$ mode described as a damped harmonic oscillator with a central frequency of $43 \mathrm{~cm}^{-1}$ [129]. THz spectroscopy of ethylammonium nitrate indicates a fast process described by a slightly underdamped harmonic oscillator with a damping time constant of $2 / \gamma=830 \mathrm{fs}$, corresponding to the typical time scale expected for an intermolecular mode, due to 
hydrogen bond reformation. ILs are understood as liquids consisting entirely of ions and having melting points below $373 \mathrm{~K}$. The presence of hydrogen bonding in the structure of an IL, 1-alkyl-3-methylimidazolium salt, was first reported by Seddon et al. in 1986 [130]. It is assumed that hydrogen bonding plays an important role in the properties and reaction dynamics of these Coulomb systems. The direct observation of hydrogen-bond stretching frequencies are reported from the FIR spectrum in pure imidazolium ILs containing the same anion, bis(trifluoromethylsulfonyl)imide $\left(\mathrm{NTf}_{2}{ }^{-}\right)$, but various cations: 1,2,3-trimethylimidazolium (1,2,3-trimethyl-im $\left.{ }^{+}\right)$, 1,3-dimethylimidazolium (1,3-dimethyl-im $\left.{ }^{+}\right), \quad$ 1,2-dimethylimizazolium $\quad\left(1,2\right.$-dimethyl-im $\left.{ }^{+}\right)$and 1-methylimidazolium $\left(1-\right.$ methyl-im ${ }^{+}$) [131]. The maximum intensities below $150 \mathrm{~cm}^{-1}$ are measured at $62.3 \mathrm{~cm}^{-1}$ (1,2,3-trimethyl-im $\left.{ }^{+}\right), 85.7 \mathrm{~cm}^{-1}\left(1,3\right.$-dimethyl-im $\left.{ }^{+}\right), 96.7 \mathrm{~cm}^{-1}\left(1,2\right.$-dimethyl-im $\left.{ }^{+}\right)$and $100.7 \mathrm{~cm}^{-1}$ (1-methyl-im ${ }^{+}$). These contributions are assigned to the stretching vibrational bands of hydrogen bonds ${ }^{+} \mathrm{C}-\mathrm{H} \cdots \mathrm{A}^{-}$and/or ${ }^{+} \mathrm{N}-\mathrm{H} \cdots \mathrm{A}^{-}$. The shift of these maxima to higher wavenumbers suggests enhanced interaction energy between the anion and cation. The stronger the hydrogen bond, the larger the wavenumber and the corresponding intensity of the hydrogen bond stretching vibrational band. In the FIR spectra of imidazolium-based ILs 1-ethyl-3-methylimidazolium bis(trifluoromethylsulfonyl)imide $\left(\left[\mathrm{C}_{2} \mathrm{mim}\right]\left[\mathrm{NTf}_{2}\right]\right), \quad$ 1-ethyl-3-methylimidazolium ethylsulfate $\left(\left[\mathrm{C}_{2} \mathrm{mim}\right]\left[\mathrm{EtSO}_{4}\right]\right)$, 1-ethyl-3methylimidazolium dicyanamide $\left(\left[\mathrm{C}_{2} \mathrm{mim}\right]\left[\mathrm{N}(\mathrm{CN})_{2}\right]\right)$ and 1-ethyl-3-metylimidazolium thiocyanate $\left(\left[\mathrm{C}_{2} \mathrm{mim}\right][\mathrm{SCN}]\right)$, the wavenumber and intensity of the absorption maximum both decrease in the order $\mathrm{SCN}^{-}, \mathrm{N}(\mathrm{CN})_{2}{ }^{-}, \mathrm{EtSO}_{4}^{-}$and $\mathrm{NTf}_{2}{ }^{-}$[132]. Though it has been argued that significant vibrational contributions may occur between two and $50 \mathrm{~cm}^{-1}$, the THz spectra of these ILs show no contribution blow $50 \mathrm{~cm}^{-1}$ [133]. The importance of hydrogen bonding in imidazolium-based ILs was the subject of highly controversial discussions. Because ILs solely exist as anions and cations, it is generally assumed that Coulomb interactions absolutely dominate the properties of these materials. Although the local and directional type of interaction contributes only one tenth of the overall interaction energy, hydrogen bonds have a significant influence on the structure of these Coulomb systems. With increasing hydrogen bond abilities and strengths, the $\mathrm{NTf}_{2}^{-}$anion is found in the cis configuration. Switching off these local interactions leads to the energetically favored trans conformation [134].

\section{Computational Methods to Analyze the THz Spectra of Hydrogen-Bonded Molecules}

The THz spectra of hydrogen-bonded molecules have a lot of peaks in the narrow spectral region of 30-200 $\mathrm{cm}^{-1}$. The assignment of peaks requires a precise DFT or first-principles calculation at an accuracy of less than $10 \mathrm{~cm}^{-1}$ in frequency. Powdered samples are popularly used for $\mathrm{THz}$ measurements, and solid-state calculations are basically required. In some cases, the gas-phase calculations ensure the analysis. In this section, we indicate the least requirement of the calculation level for the analysis of the $\mathrm{THz}$ spectra of hydrogen-bonded molecules. We do not cover all the methods in detail. The calculation level corresponding to what is stated here is needed for the reliable analysis of the $\mathrm{THz}$ spectra. Additional crucial contributions to the molecular structure and $\mathrm{THz}$ spectrum of hydrogen-bonded molecules, such as dispersive interactions and anharmonic force fields, are described in the next section.

An ab initio molecular orbital method at the MP2 (second-order Møller-Plesset perturbation theory) level and DFT calculations by different functionals (B3LYP (Becke's three parameter exchange and 
Lee-Yang-Parr correlation functional), PBE (gradient-corrected correlation functional of Perdew, Burke and Ernzerhof) and MPWB1K (a combination of a modified Perdew-Wang exchange functional by Adamo and Barone and the B1K correlation functional formulated by Truhlar and co-workers) [135]) are very useful for assigning the vibrational absorption of gas-phase molecules. It is very well known that DFT, especially with hybrid functionals, such as B3LYP, allows for an accurate prediction of the properties of hydrogen-bonded complexes. A newly developed exchange-correlation functional, MPWB1K, is reliable for predicting intermolecular vibrations of doubly hydrogen-bonded complexes, such as DNA (deoxyribonucleic acid) base pairs [136,137]. Vibrational frequency analyses should be carried out at the fully optimized geometry and at the calculation level used in the geometry optimization. To obtain a reliable frequency value, larger basis sets, such as 6-311++G(d,p) (split-valence triple-zeta polarized basis set with diffuse functions) and cc-pVTZ, are recommended. Diffuse functions are more crucial than the use of large correlation consistent basis sets, like cc-pVTZ. Vibrational frequencies, for example, calculated at the B3LYP/6-311++G(d,p) level, produced better agreement with experimental mid-IR results than the calculated frequencies at the B3LYP/cc-pVTZ level $[138,139]$. The use of a correlation consistent basis set augmented with diffuse functions, such as aug (augmented)-cc-pVTZ, would give much better results, but great computer resources are required to complete the calculation.

Frequency calculations at the B3LYP level using the 6-31+G(d) basis set gives sufficient results for large cluster systems, like ionic liquids [120,126,134]. Here, the anharmonicity described in the next section is corrected by the standard scaling factor, 0.96, and Grimme's dispersion-corrected method (DFT-D3), described in the next section, is recommended for the calculation of THz vibrations.

The total-energy calculation of the periodic system based on the plane-wave DFT method for the generalized gradient approximation (GGA) using several exchange-correlation functionals is used to investigate intermolecular vibrations in crystals. Extremely accurate calculations are necessary to clear up uncertainties with regard to the assignment of the complicated $\mathrm{THz}$ spectrum. The PBE functional is frequently selected for use, as the functional has been shown consistently to produce high-quality simulation of the solid-state structure and THz spectrum [140]. The kinetic energy cutoff, to which the valence electron wave function is expanded, is desired to be as large as possible. Electronic minimization needs to be converged to the limit of machine accuracy. Full geometry optimization for both the cell parameters and atomic coordinates is preferred. The total energy of the system needs to be converged to better than $0.1 \mathrm{meV} /$ atom in terms of k-point sampling for the integration over the Brillouin zone.

\section{Dispersive Interactions and Anharmonicity}

The THz spectrum reflects a weak intermolecular interaction, and thus, the dispersive interactions and anharmonic force fields play a decisive role in the peak assignment. One of the critical issues in molecular-crystals simulation is the proper description of noncovalent interactions between molecules (i.e., hydrogen bonding, $\mathrm{vdW}$ interactions, dipolar effects, etc.) that dictate the crystalline structure and the thermodynamic properties controlling phase transitions. In particular, dispersion correction is important for weak hydrogen bond networks in crystals. It is generally accepted that the electrostatic 
and induction components are smaller in weak hydrogen bonds and that the dispersive interaction is relatively larger $[49,50,141]$.

The high level of quantum chemical methods (e.g., a coupled cluster with single, double and triple perturbative excitations, $\operatorname{CCSD}(\mathrm{T})$ ) can attain a consistently accurate description of vdW interactions. Unfortunately, $\operatorname{CCSD}(\mathrm{T})$ is limited to rather small systems, due to its high computational cost and steep $\mathrm{O}\left(\mathrm{N}^{7}\right)$ scaling with system size. MP2 is the most economical wave-function-based electronic structure method beyond the Hartree-Fock (HF) approximation, which provides an approximate description of all relevant $\mathrm{vdW}$ interactions-electrostatics, induction and dispersion. One of the serious shortcomings of the MP2 theory is the noticeable overestimation of dispersion-interaction energy $[142,143]$. DFT is widely used in the computationally chemistry community, and B3LYP is the most popular density functional. However, one of the serious shortcomings of B3LYP is the inaccuracy of interactions dominated by medium-range correlation energy, such as vdW attraction. The Minnesota 05 (M05) and Minnesota 06 (M06) classes of functionals developed by the group of Truhlar at the University of Minnesota are new hybrid meta-GGA exchange-correlation functional [144-146] with broad applicability in chemistry. Based on the comparison of the performance among M06 class functionals and one M05 class functional (M05-2X) for diverse databases, M06-2X, M05-2X, M06-HF, M06 and M06-L are recommended for the study of noncovalent interactions [147]. M06-L is local functional with 0\% HF exchange. M05, M05-2X, M06, M06-2X, M06-HF are global hybrid functionals with 26\%-100\% HF exchange. The structure and vibrational properties of the hydrogen-bonded molecules, complexes and clusters are studied with the use of M06 class functionals [148-159].

It is well known that DFT does not describe a noncovalent interaction, though DFT provides reasonably accurate predictions for many properties of various molecules and solids and is the most widely used method for electronic structure calculations in condensed matter physics and quantum chemistry [160-162]. The development of procedures for dispersion correction to DFT is an active area of research. Among the most popular methods are the DFT-D2 [163] and DFT-D3 [164] procedures by Grimme and co-workers. The dispersion-correction term is given by Equation (1):

$$
-\sum_{n=3}^{\infty} f_{2 n}(R)\left(C_{2 n} / R^{2 n}\right)
$$

Here, $R$ is an interatomic distance, $f$ denotes a dumping function and $C$ is a dispersion coefficient. In the DFT-D2 approach, dispersion corrections are introduced by adding the first damped atom-atom $C_{6} / R^{6}$ term of Equation (1), and in the DFT-D3 approach, they are incorporated through the first two damped atom-atom $C_{6} / R^{6}$ and $C_{8} / R^{8}$ terms of Equation (1). These terms are smoothly cut off in the short range, where they are not relevant, but explicitly enforce the desired long-range asymptotic behavior. In the DFT-D2 method, the parameters are obtained by fitting to experimental $C_{6}$ coefficients and/or post-Hartree-Fock binding energy data and are independent of the electronic structures and chemical environments. This deficiency is partially remedied in DFT-D3, where the $C_{6}$ coefficients depend on the coordination number of atoms. Of the closely related approaches, the vdW-TS (Tkatchenko-Scheffler) approach $[165,166]$ is particularly intriguing because of how the $C_{6}$ coefficients depend on the chemical environment. The vdW-TS approach is a parameter-free method for the accurate determination of long-range vdW interactions from mean-field electronic structure 
calculations and improves the description of weakly bonded systems in DFT for a range of exchange correlation functionals. The utility of the vdW-TS procedure has been explored for describing several crystalline materials, including noble gases, molecular crystals and layered materials, in which dispersion interactions are important [167].

The calculation of vibrational frequencies is performed at the minimum on the potential energy surface. Two alternatives in the geometry optimization process are open for including a dispersive interaction into solid-state DFT (or first-principles) calculations (Figure 7). Crystallographic data in CIF (Crystallographic Information File) format are useful as the initial structure for geometry optimization. Full optimization is basically preferred (Process A of Figure 7). Full optimization means both the cell parameters and atomic coordinates are optimized. When dispersion correction is not included in DFT or the first-principles, fixed cell parameters or fixed atomic coordinates at the experimental values are frequently used to play the role of the dispersion force (Process B of Figure 7), though this is not true dispersion correction. The reasonable performance of the latest dispersion-corrected DFT has been demonstrated for systems containing weak hydrogen bonds [168]. Potential energy curves for five complexes with weak to medium strong hydrogen bonds have been computed with dispersion-corrected DFT methods. Dispersion-corrected DFT in two totally different approaches (density based and pair-wise corrected) is able to provide a rather accurate and consistent description of weakly hydrogen-bonded systems compared to the high level of $a b$ initio calculations. The typical deviation for intermolecular distances is $0.05 \AA$ and the binding energies are accurate within about $5 \%-10 \%$.

Figure 7. Two alternatives $(\mathbf{A}, \mathbf{B})$ in the geometry optimization process for incorporating a dispersive interaction into first-principles calculations.

$\underline{\text { Initial structures for geometry optimization }}$ (Crystallographic data in CIF (Crystallographic Information File) format is useful.)

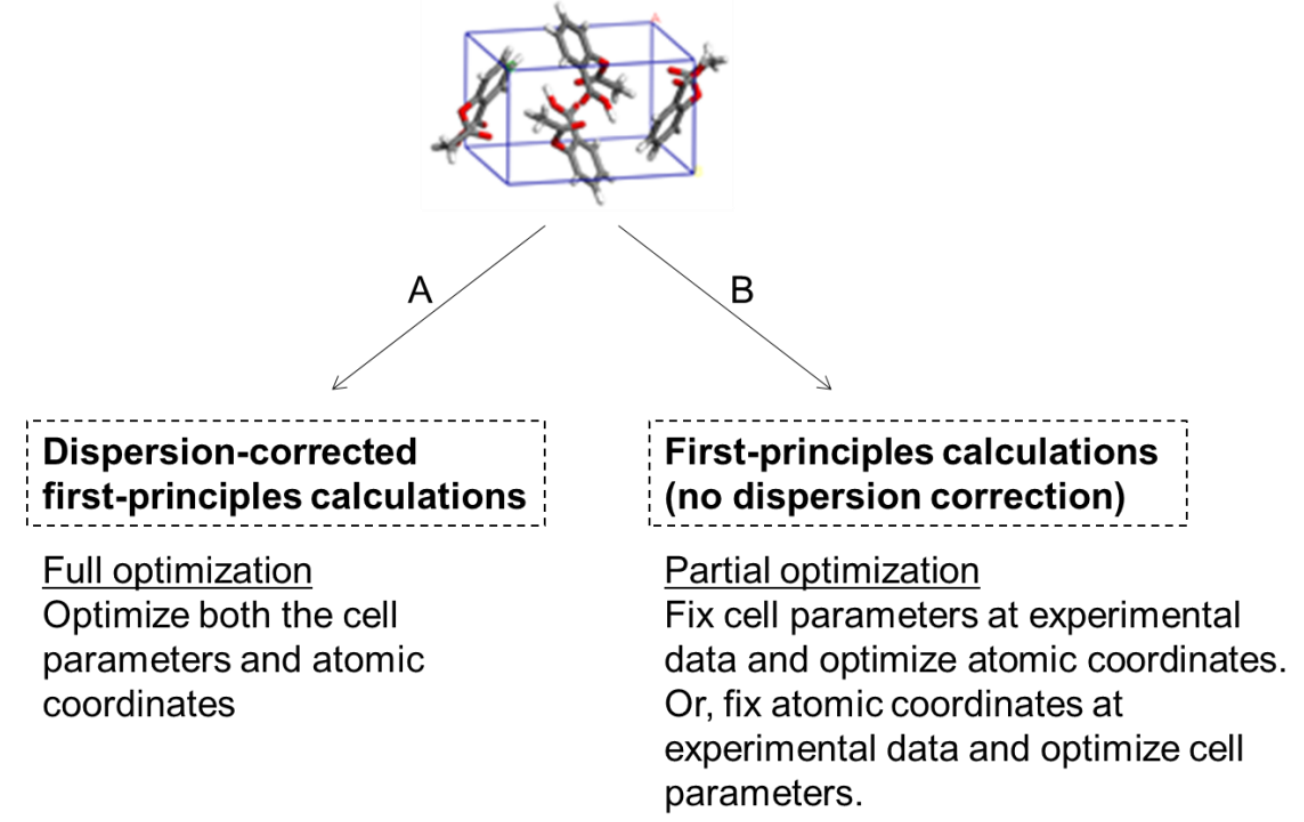

The dispersion-corrected first-principles calculation using the Grimme method [163] and the refinement by Civalleri et al. [169] has been performed intensively by Korter and co-workers over a 
couple of years to analyze the THz spectra [170-172] (Process A of Figure 7). The effects of applying an empirical dispersion correction to solid-state DFT methods were evaluated in the simulation of the crystal structure and low-frequency $\mathrm{THz}$ spectrum of naproxen, the non-steroid anti-inflammatory drug. Inclusion of a modified dispersion correction enabled a high-quality simulation of the $\mathrm{THz}$ spectrum and the crystal structure of naproxen to be achieved without the need for artificially constraining the unit cell dimensions. The dispersion contributions calculated using existing published parameters were, however, too large for the accurate reproduction of the experimental crystal structure and $\mathrm{THz}$ spectrum. In comparison with the empirical dispersion correction by the Grimme approach and the refinement by Civalleri, the use of fixed lattice dimensions obtained from experimental measurements in the DFT calculations (Process B of Figure 7) is an alternative that results in high-quality simulations of the $\mathrm{THz}$ spectra. The dispersion-corrected first-principles calculation using the vdW-TS approach has been performed very recently to assign the $\mathrm{THz}$ spectrum of weakly hydrogen-bonded systems $[72,173]$. Full geometry optimization has been performed for both the atomic coordinates and lattice parameters (Process A of Figure 7). Unit-cell parameters are properly converged with a reduction of $1 \%-2 \%$ from experimental values. The results are consistent with the thermal expansion that would occur upon heating from $0 \mathrm{~K}$ (first-principles calculations) to a finite temperature (experiments) [174]. The calculated frequencies and the relative intensities excellently reproduce the observed THz spectrum, where no scaling factor is applied to the calculated frequency values. The deviation in frequency is a few percent normally. Some torsion modes and translational modes show relatively large deviations, but the difference from experimental values is approximately $10 \mathrm{~cm}^{-1}$ and is acceptable for the accuracy for the spectral assignment.

The frequency of harmonic vibrations overestimates the experimental fundamental frequencies at the $a b$ initio self-consistent-field (SCF) level of theory [175-178], due to basis-set incompleteness, electron correlation effects and anharmonicity. Nowadays, the basis set converges nearly to completeness, and the electron correlation is incorporated properly by several methods. Even with the use of a basis set large enough and the adequate incorporation of electron correlations, there remains a deviation attributed to anharmonicity. Several scaling factors were proposed for harmonic frequencies obtained at different levels of $a b$ initio, DFT and semiempirical methods [179]. The deviation of the potential energy well of a simple harmonic oscillator (Figure 8a) from the actual potential energy well of an anharmonic oscillator (Figure $8 \mathrm{~b}$ ) is compensated for by scaling factors smaller than 1.0. The simple harmonic oscillator has a potential energy well that is parabolic in shape, symmetrical about the equilibrium bond length. The potential energy function contains a single quadratic term. When treaded with an anharmonic oscillator, the potential energy well is asymmetric, and dissociation can occur. The potential energy function contains asymmetric cubic, quartic, etc., terms. If one assumes that the form of the potential energy curve is that defined by the Morse potential, this is a good, although not a perfect, approximation to the real potential. As can be seen from the shape of the potential, the deviation from the Morse potential is large at the high-frequency end, and monotonic scaling by a factor is rational. 
Figure 8. (a) The parabolic potential energy as a simple harmonic oscillator and (b) the actual potential energy - an anharmonic oscillator.
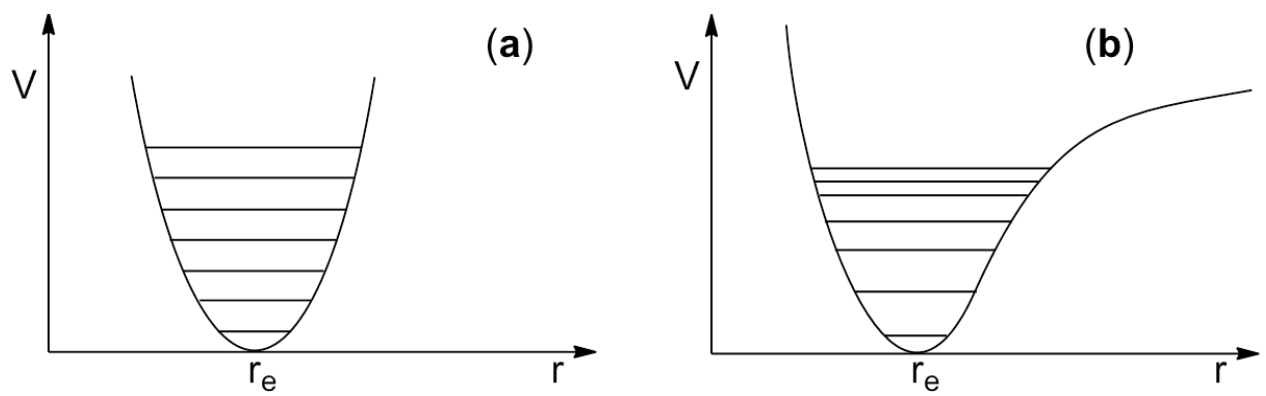

Significant and systematic overestimations of the predicted vibrational frequencies were observed in solid-state calculations of co-crystallized $\mathrm{H}_{2} \mathrm{O}$ molecules with a small biomolecule, L-serine [180]. Evidence provided by the comparison of the experimental and calculated vibrational frequencies for both the protonated and deuterated L-serine $\cdot \mathrm{H}_{2} \mathrm{O}$ solids indicates the presence of significant anharmonicity in the observed lattice vibrations. Vibrational anharmonicity may actually play a much larger role in the interpretation of the $\mathrm{THz}$ spectra of hydrates in contrast to their corresponding anhydrous forms. The incorporation of water molecules into the crystal structure of pharmaceuticals must be monitored, since the hydrate form can be easily created or destroyed during the manufacturing process, and the crystalline hydrate often has drastically different physical properties in comparison to the anhydrous form [181].

The scaling factor to compensate for the overestimation of the predicted vibrational frequencies of the harmonic approximation is a few percent less than 1.0 at the latest high-level of quantum chemical calculation. By applying the scaling factor to the calculated harmonic frequencies in the $\mathrm{THz}$ region, the value shifts by a few $\mathrm{cm}^{-1}$. Anharmonic vibrational spectra, including the $\mathrm{THz}$ region of sulfuryl halides $\mathrm{SO}_{2} \mathrm{X}_{2}(\mathrm{X}=\mathrm{F}, \mathrm{Cl}, \mathrm{Br})$ [182] and hydrogen-bonded systems [183], have been computed by the $a b$ initio or first-principles calculations using the vibrational self-consistent field (VSCF) method. The anharmonic frequencies are lowered in the mid-IR region, compared with harmonic frequencies. In the $\mathrm{THz}$ region, the anharmonic frequencies are red-shifted in $\mathrm{SO}_{2} \mathrm{X}_{2}$, but those are rather blue-shifted in the hydrogen-bonded systems. By the finite displacement procedure, the anharmonic infrared spectrum of $\mathrm{ClONO}_{2}$ and $\mathrm{BrONO}_{2}$, including the $\mathrm{THz}$ region, has been computed [184]. The anharmonic frequency shows a blue shift in the $\mathrm{THz}$ region, although there is a red shift in the mid-IR region. Calculated anharmonic vibrational frequencies of a benzoic acid dimer linked by two hydrogen bonds also gives both red and blue shifts in the THz region compared with the harmonic frequencies [119]. Complicated potential wells seem to cause the anharmonicity in the low-frequency region, such as a double-well potential with a very low energy barrier and a very flat energy potential that causes a large amplitude oscillation.

\section{Temperature-Dependent THz Spectrum}

The temperature dependence of the $\mathrm{THz}$ spectrum allows access to vital information about the structure and vibrational dynamics of solids. Drastic temperature dependences are frequently observed in the $\mathrm{THz}$ spectral region, while in some cases, the $\mathrm{THz}$ spectra are insensitive to temperature. 
Hydrogen-bonded networks are easily broken compared to covalent bonds and facilitate structural changes at room temperature (Figure 9) [185]. The stretching vibration of weaker kinds of hydrogen bonds can be detected only at low temperatures. Among the spectral changes of vibrational absorptions by temperature, polarization wave, isotope, and so on, the temperature dependence is the most effective for revealing the weak intermolecular interaction and global nuclear motion appearing in the $\mathrm{THz}$ spectral region. There is quite a good possibility that the temperature dependence of the $\mathrm{THz}$ spectra is related to the formation of weak bonds.

Figure 9. The temperature-dependent behavior of weak bonds in crystals.
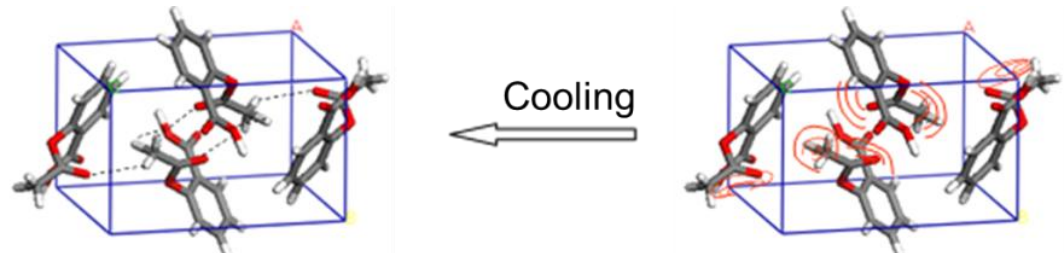

The decrease of temperature causes an increase of the number of peaks, a shift of the frequency values, a reduction of the peak width, and so on, in the THz spectra. A number of vibrational modes of hydrogen-bonded purine and adenine were found to shift to higher intensities and frequencies in the FIR vibrational spectra using THz-TDS, as the temperature was reduced [186]. Nucleic acid bases are the building blocks of DNA and ribonucleic acid (RNA), and there have been numerous experimental investigations of nucleic acid bases and nucleosides using mid-IR and Raman spectroscopy, as well as neutron inelastic scattering [187-190]. Adenine is one of two common purine bases and is found in both DNA and RNA. The intermolecular hydrogen-bonding signature of self-assembled phospholipids was studied by means of $\mathrm{THz}$ spectroscopy, and significant information at the molecular level has been provided [191]. Phospholipid layers allow the monitoring of the IR spectrum via well-defined assays and via the temperature-dependent phase transition coupled to specific intra- and intermolecular hydrogen bonding and to the interaction with water. The signals typical for bound water in the mid-IR region showed an analogous temperature dependence for the mixed and pure lipids, whereas the hydrogen-bonding features in the $\mathrm{THz}$ region showed a very different behavior for the two types of lipid films. The contribution of water molecules does not exclusively dominate the spectral range in the $\mathrm{THz}$ region for this type of film (or at least, it is only one of the possible contributions), and any type of hydrogen bonding may contribute.

Larger molecules sometimes show complicated $\mathrm{THz}$ spectra even at room temperature. Furthermore, the peak splitting due to crystal packing forces, the shift of wavenumbers and the sharpening of peaks occur at low temperature. The gas-phase calculation of such a large molecule can be an alternative to the solid-state calculation in assigning the room-temperature spectrum, if solid-state calculations are difficult to perform with reliable accuracy, due to the insufficient capacity of computer power. The measurement of related molecules that are a part of a large molecule is informative for the peak assignment by the gas-phase calculation. Riboflavin consists of a ring part and a side chain. The set of related compounds are alloxazine and lumichrome for the ring part and D-mannitol for the side chain (Figure 10). A strong peak was observed at $99.5 \mathrm{~cm}^{-1}$ at room temperature in the $\mathrm{THz}$ spectrum of riboflavin, and the band has a shape and temperature-dependent behavior similar to the band of 
D-mannitol at $96.6 \mathrm{~cm}^{-1}$. The strong peak of riboflavin at $99.5 \mathrm{~cm}^{-1}$ is assigned to the torsion mode of the ribityl group with the gas-phase calculations [139]. Disaccharide, $\alpha, \alpha$-trehalose dihydrate has two water molecules embedded in the molecule. Two distinct peaks were observed in the room-temperature $\mathrm{THz}$ spectrum; those are assigned to the torsion mode of the hydroxymethyl group linked to the hydrogen atoms of two water molecules with the gas-phase calculation [192].

Figure 10. Riboflavin and related molecules.

\section{Ring part}
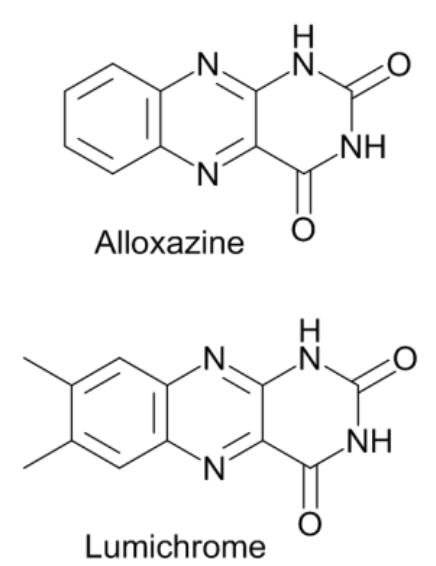

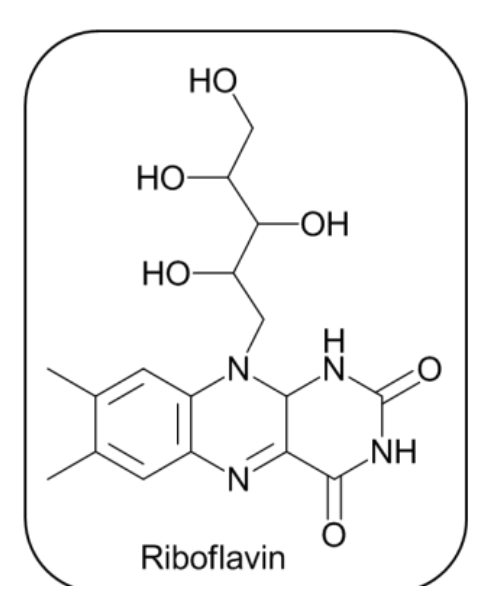

Side chain

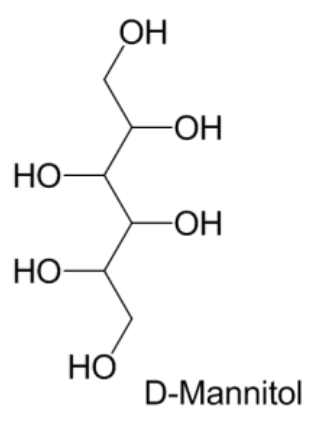

Translational vibrations between molecules are clearly observed at low temperature, and the stretching-vibration mode of the weak hydrogen bond is mixed in the translational vibration. The difference in the network of weak hydrogen bonds is reflected in the low-temperature $\mathrm{THz}$ spectrum. Benzoic acid, salicylic acid and acetylsalicylic acid (aspirin) have different networks of weak hydrogen bonds in crystals. Those molecules have a different substituent at the ortho position. All of the three compounds take a centrosymmetric dimer in crystals, but the networks of their weak hydrogen bonds completely differ from each other. Benzoic acid has a layered structure. Salicylic acid takes on a non-layered structure, and aspirin forms a one-dimensional-chain structure sustained by alternating the carboxylic acid and acetyl groups. The stretching vibration of the weak hydrogen bond calculated with the solid-state DFT demonstrates the respective bonding. The revealed stretching vibrations of weak hydrogen bonds between molecules are absent in benzoic acid; there are three in salicylic acid and one in aspirin [72,119,173].

\section{Polarization-Dependent THz Spectrum}

The polarization-dependent $\mathrm{THz}$ spectrum determines the orientation of the vibrational dipole moments. The $\mathrm{THz}$ absorption spectrum of powdered samples reflects the average property of randomly orientated molecules. On the other hand, the $\mathrm{THz}$ spectrum of single crystals shows the polarization and/or angle dependence [192-195]. Another approach to detect the polarization dependence of the $\mathrm{THz}$ spectrum is the measurement of polycrystalline thin film, which is planar-ordered relative to the metal plate and, hence, relative to the THz polarization [196-198].

Several reports have been given on the polarization dependence at the low-frequency region below $100 \mathrm{~cm}^{-1}$. The polarization dependence was observed both for the sharp and broad peaks. The FIR spectrum of crystalline $\alpha, \alpha$-trehalose dihydrate measured at $4 \mathrm{~K}$ with a polarized $\mathrm{THz}$ wave showed a 
clear polarization dependence, especially in the sharp peaks below $60 \mathrm{~cm}^{-1}$ [192]. The angle-dependent THz spectra of the L-cysteine and L-histidine single crystals measured with THz-TDS indicated a clear hydrogen-bond peak as a result of the hydrogen-bonded network [193]. The powder spectra measured at low temperature show more intermolecular vibrational modes than those measured at room temperature. The FIR spectra of (100), (010) and (001)-oriented 1,3,5-trinitro- $S$-triazine single crystals showed a number of discrete absorptions ranging from 10 to $100 \mathrm{~cm}^{-1}$, and the high dependence of absorption on the orientation of the THz polarization with respect to the crystallographic axis [194]. The polarization-dependent spectra of a stretched polyhydroxybutyrate crystal determined the direction of the vibrational transition moments [195]. The interpretation of the observed polarization dependences is not sufficient without a spectral analysis by solid-state calculations. The spectral change of planar-ordered samples in the measurements with narrow-line waveguide THz-TDS by Grischkowsky and co-workers is well correlated with the direction of the vibrational motion with the precise first-principles calculations (Figure 11) [192,196-198].

Figure 11. Displacement vectors of the decreasing and unchanged modes with a film of aspirin [192].

\section{Decreasing modes with a film}
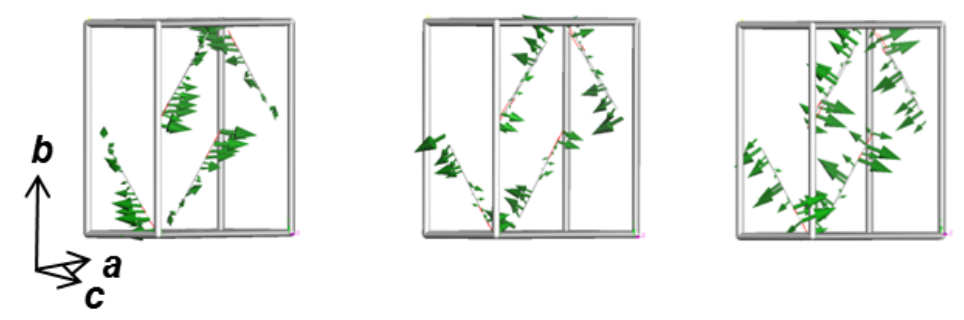

Unchanged modes with a film
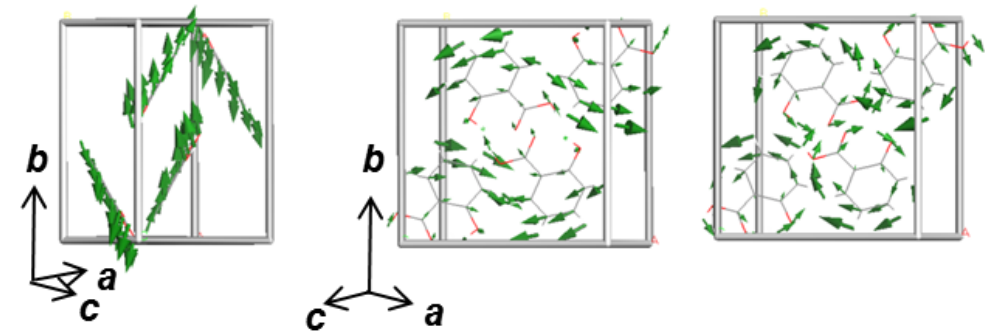

\section{Conclusions}

$\mathrm{THz}$ vibrations are a suitable indicator for the detection of weak bonding in crystals. Though FIR spectroscopy, synonymous with THz spectroscopy, first emerged in 1892, the spectral research in this area did not progress much, due to the broadness and weakness of the peaks in this spectral region. In addition, computational codes to analyze the spectrum were incomplete until very recently. With the recent development of $\mathrm{THz}$ technology and an efficient computational code, $\mathrm{THz}$ spectroscopy has attained a position as a tool for clarifying the formation of weak bonds in crystals. The THz spectra of several materials have been accumulating day by day. From the experimental point of view, the temperature and polarization dependence provide additional information about the peak characterization. From a theoretical point of view, the incorporation of dispersive interaction and 
anharmonicity into the solid-state first-principles (or DFT) calculations increases the accuracy of the assignment and avoids uncertainty.

Molecular crystals are assembled with noncovalent packing forces. The packing force is very weak, but the assembled crystal shows an identical structure or, at most, the difference of polymorphs with different network patterns. The stretching vibration of weak hydrogen bonds has long been expected to be in the $\mathrm{THz}$ region. Explicit reference to the stretching vibration has just begun, since appropriate dispersive interactions have been incorporated into the first-principles or DFT calculations very recently. Dispersive interaction is not incorporated in pure DFT, but is crucial in weak hydrogen bonds, which are at the vdW end of the types of hydrogen bonds. Due to the development of computational codes, including dispersive interactions, lattice parameters after full optimization deviate by less than $1 \%$ from crystallographic data. The effect of dispersive interactions on the frequency calculation of $\mathrm{THz}$ vibrations is significant, and thus, the incorporation of dispersive interactions into the calculation of $\mathrm{THz}$ vibrations is unavoidable for obtaining a clear assignment. Experimental and theoretical techniques have reached the point of applying $\mathrm{THz}$ spectroscopy to the areas of medicine, pharmacy, agronomy, and so on, beyond basic research.

\section{Acknowledgments}

The author acknowledges the support by the Ministry of Education, Culture, Sports, Science and Technology of Japan (grant No. 22550003).

\section{Conflicts of Interest}

The author declares no conflict of interest.

\section{References}

1. Mori, Y.; Takahashi, Y.; Iwai, T.; Yoshimura, M.; Yap, Y.K.; Sasaki, T. Slope nucleation method for the growth of high-quality 4-dimethylamino-methyl-4-stilbazolium-tosylate (DAST) crystals. Jpn. J. Appl. Phys. 2000, 39, L1006-L1008.

2. Zheng, X.; McLaughlin, C.V.; Cunningham, P.; Hayden, L.M. Organic broadband terahertz sources and sensors. J. Nanoelectron. Optoelectron. 2007, 2, 58-76.

3. Paul, O.; Beigang, R.; Rahm, M. Highly selective terahertz bandpass filters based on trapped mode excitation. Opt. Express 2009, 17, 18590-18595.

4. Theuer, M.; Beigang, R.; Grischkowsky, D.R. Adiabatic compression of terahertz waves using metal flares. Appl. Phys. Lett. 2010, 96, 191110:1-191110:3.

5. Mendis, R.; Nag, A.; Chen, F.; Mittleman, D.M. A tunable universal terahertz filter using artificial dielectrics based on parallel-plate waveguides. Appl. Phys. Lett. 2010, 97, 131106:1-131106:3.

6. Mendis, R.; Mittleman, D.M. A 2-D artificial dielectric with $0<n<1$ for the terahertz region. IEEE Trans. Microw. Theory Tech. 2010, 58, 1993-1998.

7. Nagai, M.; Mukai, N.; Minowa, Y.; Ashida, M.; Takayanagi, J.; Ohtake, H. Achromatic THz wave plate composed of stacked parallel metal plates. Opt. Lett. 2014, 39, 146-149. 
8. Theuer, M.; Torosyan, G.; Ellrich, F.; Jonuscheit, J.; Beigang, R. Terahertz imaging in industrial applications. TM Tech. Mess. 2009, 75, 64-70.

9. Mittleman, D.M.; Jacobsen, R.H.; Neelamani, R.; Baraniuk, R.G.; Nuss, M.C. Gas sensing using terahertz time-domain spectroscopy. Appl. Phys. B 1998, 67, 379-390.

10. Zandonella, C. Terahertz imaging: T-ray specs. Nature 2003, 424, 721-722.

11. Federici, J.F.; Schulkin, B.; Huang, F.; Gary, D.; Barat, R.; Oliveira, F.; Zimdars, D. THz imaging and sensing for security applications-Explosives, weapons and drugs. Semicond. Sci. Technol. 2005, 20, S266-S280.

12. Harsha, S.S.; Melinger, J.S.; Qadri, S.B.; Grischkowsky, D. Substrate independence of THz vibrational modes of polycrystalline thin films of molecular solids in waveguide THz-TDS. $J$. Appl. Phys. 2012, 111, doi:10.1063/1.3678000.

13. Yang, Y.; Harsha, S.S.; Shutler, A.J.; Grischkowsky, D.R. Identification of Genistein and Biochanin A by THz (far-infrared) vibrational spectra. J. Pharm. Biomed. Anal. 2012, 62, 177-181.

14. Koch, M. Terahertz communications: A 2020 vision. In Terahertz Frequency Detection and Identification of Materials and Objects (NATO Science for Peace and Security Series); Miles, R.E., Zhang, X.-C., Eisele, H., Krotkus, A., Eds.; Springer: Dordrecht, The Netherlands, 2007; Theme 4, pp. 325-338.

15. Yang, Y.; Mandehgar, M.; Grischkowsky, D.R. Broadband THz pulse transmission through the atmosphere. IEEE Trans. THz Sci. Technol. 2011, 1, 264-273.

16. Koenig, S.; Lopez-Diaz, D.; Antes, J.; Boes, F.; Henneberger, R.; Leuther, A.; Tessmann, A.; Schmogrow, R.; Hillerkuss, D.; Palmer, R.; et al. Wireless sub-THz communication system with high data rate. Nat. Photon. 2013, 7, 977-981.

17. Mandehgar, M.; Yang, Y.; Grischkowsky, D. Atmosphere characterization for simulation of the two optimal wireless terahertz digital communication links. Opt. Lett. 2013, 38, 3437-3440.

18. Ho, L.; Pepper, M.; Taday, P. Terahertz spectroscopy: Signature and fingerprints. Nat. Photon. 2008, 2, 541-543.

19. Ueno, Y.; Ajito, K. Analytical terahertz spectroscopy. Anal. Sci. 2008, 24, 185-192.

20. Davies, A.G.; Burnett, A.D.; Fan, W.; Linfield, E.H.; Cunningham, J.E. Terahertz spectroscopy of explosives and drugs. Mater. Today 2008, 11, 18-26.

21. McGoverin, C.M.; Rades, T.; Gordon K.C. Recent pharmaceutical applications of Raman and terahertz spectroscopies. J. Pharm. Sci. 2008, 97, 4598-4621.

22. Smith, R.M.; Arnold, M.A. Terahertz time-domain spectroscopy of solid samples: Principles, applications, and challenges. Appl. Spectrosc. Rev. 2011, 46, 636-679.

23. Theuer, M.; Harsha, S.S.; Molter, D.; Torosyan, G.; Beigang, R. Terahertz time-domain spectroscopy of gases, liquids, and solids. ChemPhysChem 2011, 12, 2695-2705.

24. McIntosh, A.I.; Yang, B.; Goldup, S.M.; Watkinson, M.; Donnan, R.S. Terahertz spectroscopy: A powerful new tool for the chmical sciences? Chem. Soc. Rev. 2012, 41, 2072-2082.

25. Sushko, O.; Dubrovka, R.; Donnan, R.S. Analysis of optical thickness determination of materials by THz-TDS. J. Phys. Conf. Ser. 2013, 472, doi:10.1088/1742-6596/472/1/012005.

26. Ji, T.; Zhang, Z.; Zhao, H.; Chen, M.; Yu, X.; Xiao, T. A THz-TDS measurement method for multiple samples. Opt. Commun. 2014, 312, 292-295.

27. Hübers, H.-W. Towards THz integrated photonics. Nat. Photon. 2010, 4, 503-504. 
28. Ferguson, B.; Zhang, X.-C. Materials for terahertz science and technology. Nat. Mater. 2002, 1, 26-33.

29. Miles, R.; Harrison, P.; Lippens, D. Terahertz Sources and Systems; NATO Science Series II; Kluwer: Dordrecht, The Netherlands, 2001.

30. Siegel, P.H. Terahertz technology. IEEE Trans. Microw. Theory Tech. 2002, 50, 910-928.

31. Beard, M.C.; Turner, G.M.; Schmuttenmaer, C.A. Terahertz spectroscopy. J. Phys. Chem. B 2002, 106, 7146-7159.

32. Hangyo, M.; Tani, M.; Nagashima, T. Terahertz time-domain spectroscopy of solids: A review. Int. J. Infrared Millim. Wave 2005, 26, 1661-1690.

33. Tonouchi, M. Cutting-edge terahertz technology. Nat. Photon. 2007, 1, 97-105.

34. Ellrich, F.; Weinland, T.; Theuer, M.; Jonuscheit, J.; Beigang, R. Fiber-coupled terahertz spectroscopy system. TM Tech. Mess. 2008, 75, 14-22.

35. Ellrich, F.; Klier, J.; Jonuscheit, J.; Weinland, T.; Beigang, R. Terahertz waves-A new spectral band for industrial measurement techniques. TM Tech. Mess. 2010, 77, 452-461.

36. Baxter, J.B.; Guglietta, G.W. Terahertz spectroscopy. Anal. Chem. 2011, 83, 4342-4368.

37. Wang, C.; Lu, B.; Lin, C.; Chen, Q.; Miao, L.; Deng, X.; Zhang, J. 0.34-THz wireless link based on high-order modulation for future wireless local area network applications. IEEE Trans. THz Sci. Technol. 2014, 4, 75-85.

38. Xu, H.; Wu, B.; Iverson, E.W.; Low, T.S.; Feng, M. $0.5 \mathrm{THz}$ performance of a type-II DHBT with a doping-graded and constant-composition GaAsSb base. IEEE Electron Dev. Lett. 2014, 35 , 24-26.

39. Smirnov, I.A.; Alekseev, E.A.; Ilyushin, V.V.; Margulés, L.; Motiyenko, R.A.; Drouin, B.J. Spectroscopy of the ground, first and second excited torsional states of acetaldehyde from 0.05 to 1.6 THz. J. Mol. Spectrosc. 2014, 295, 44-50.

40. Bosshard, C.; Sutter, K.; Prêtre, P.; Hulliger, J.; Flörsheimer, M.; Kaatz, P.; Günter, P. Organic Nonlinear Optical Materials (Advances in Nonlinear Optics); Gordon and Breach Science Publishers SA: Basel, Switzerland, 1995; Volume 1.

41. Wong, M.S.; Pan, F.; Gramlich, V.; Bosshard, C.; Günter, P. Self-assembly of an acentric co-crystal of a highly hyperpolarizable merocyanine dye with optimized alignment for nonlinear optics. Adv. Mater. 1997, 9, 554-557.

42. Bosshard, C.; Pan, F.; Wong, M.S.; Manetta, S.; Spreiter, R.; Cai, C.; Günter, P.; Gramlich, V. Nonlinear optical organic co-crystals of merocyanine dyes and phenolic derivatives with short hydrogen bonds. Chem. Phys. 1999, 245, 377-394.

43. Choubey, A.; Kwon, O.-P.; Jazbinsek, M.; Günter, P. High-quality organic single crystalline thin films for nonlinear optical applications by vapor growth. Cryst. Growth Des. 2007, 7, 402-405.

44. Günter, P. Nonlinear Optical Effects and Materials; Springer Series in Optical Sciences; Springer: Berlin, Germany, 2000; Volume 72.

45. Kwon, O.-P.; Jazbinsek, M.; Yun, H.; Seo, J.-I.; Kim, E.-M.; Lee, Y.-S.; Günter, P. Pyrrole-based hydrazone organic nonlinear optical crystals and their polymorphs. Cryst. Growth Des. 2008, 8, 4021-4025.

46. Kwon, S.-J.; Jazbinsek, M.; Kwon, O-P.; Günter, P. Crystal growth and morphology control of OH1 organic electrooptic crystals. Cryst. Growth Des. 2010, 10, 1552-1558. 
47. Jepsen, P.U.; Clark, S.J. Precise ab-initio prediction of terahertz vibrational modes in crystalline systems. Chem. Phys. Lett. 2007, 442, 275-280.

48. Oppenheim, K.C.; Korter, T.M.; Melinger, J.S.; Grischkowsky, D. Solid-state density functional theory investigation of the terahertz spectra of the structural isomers 1,2-dicyanobenzene and 1,3-dicyanobenzene. J. Phys. Chem. A 2010, 114, 12513-12521.

49. Steiner, T. The hydrogen bond in the solid state. Angew. Chem. Int. Ed. 2002, 41, 48-76.

50. Desiraju, G.R.; Steiner, T. The Weak Hydrogen Bond. In Structural Chemistry and Biology; Oxford University Press: Oxford, UK, 1999.

51. Jeffrey, G.A.; Saenger, W. Hydrogen Bonding in Biological Structures; Springer-Verlag: New York, NY, USA, 1991.

52. Hadži, D.; Bratos, S. Vibrational spectroscopy of the hydrogen bond. In The Hydrogen Bond: Recent Developments in Theory and Experiment; Schuster, P., Zundel, G., Sandorfy, C., Eds.; North-Holland Publishing Company: Amsterdam, The Netherlands, 1976; Volume 2, pp. 565-612.

53. Zeegers-Huyskens, T.; Huyskens, P.L. Intermolecular forces. In Intermolecular Forces; Huyskens, P.L., Luck, W.A.P., Zeegers-Huyskens, T., Eds.; Springer-Verlag: Berlin, Germany, 1991; pp. 1-30.

54. Hamilton, W.C.; Ibers, J.A. Hydrogen Bonding in Solids; W.A. Benjamin: New York, NY, USA, 1968.

55. Hadži, D. Theoretical Treatments of Hydrogen Bonding; Wiley: Chichester, UK, 1997.

56. Gilli, P.; Bertolasi, V.; Ferretti, V.; Gilli, G. Covalent nature of the strong homonuclear hydrogen bond. Study of the $\mathrm{O}-\mathrm{H} \cdots \mathrm{O}$ system by crystal structure correlation methods. J. Am. Chem. Soc. 1994, 116, 909-915.

57. Jeffrey, G.A. An Introduction to Hydrogen Bonding; Oxford University Press: Oxford, UK, 1997.

58. Sutor, D.J. The O-H $\cdots$ O hydrogen bond in crystals. Nature 1962, 195, 68-69.

59. Taylor, R.; Kennard, O. Crystallographic evidence for the existence of $\mathrm{C}-\mathrm{H} \cdots \mathrm{O}, \mathrm{C}-\mathrm{H} \cdots \mathrm{N}$, and C-H...Cl hydrogen bonds. J. Am. Chem. Soc. 1982, 104, 5063-5070.

60. Byrn, S.R.; Pfeiffer, R.R.; Stowell, J.G. Solid-State Chemistry of Drugs, 2nd ed.; SSCI, Inc.: West Lafayette, IN, USA, 1999.

61. Bernstein, J. Polymorphism in Molecular Crystals; Oxford University Press: Oxford, UK, 2002.

62. Threlfall, T.L. Analysis of organic polymorphs. A review. Analyst 1995, 120, 2435-2460.

63. Desiraju, G.R. Crystal gazing: Structure prediction and polymorphism. Science 1997, 278, 404-405.

64. Upadhya, P.C.; Nguyen, K.L.; Shen, Y.C.; Obradovic, J.; Fukushige, K.; Griffiths, R.; Gladden, L.F.; Davies, A.G.; Linfield, E.H. Characterization of crystalline phase-transformations in theophylline by time-domain terahertz spectroscopy. Spectrosc. Lett. 2006, 39, 215-224.

65. Day, G.M.; Zeitler, J.A.; Jones, W.; Rades, T.; Taday, P.F. Understanding the influence of polymorphism on phonon spectra: Lattice dynamics calculations and terahertz spectroscopy of carbamazepine. J. Phys. Chem. B 2006, 110, 447-456.

66. Nguyen, K.L.; Friščić, T.; Day, G.M.; Gladden, L.F.; Jones, W. Terahertz time-domain spectroscopy and the quantitative monitoring of mechanochemical cocrystal formation. Nat. Mater. 2007, 6, 206-209. 
67. Heinz, A.; Gordon, K.C.; McGoverin, C.M.; Rades, T.; Strachan, C.J. Understanding the solid-state forms of fenofibrate-A spectroscopic and computational study. Eur. J. Pharm. Biopharm. 2009, $71,100-108$.

68. King, M.D.; Davis, E.A.; Smith, T.M.; Korter, T.M. Importance of accurate spectral simulations for the analysis of terahertz spectra: Citric acid anhydrate and monohydrate. J. Phys. Chem. A 2011, 115, 11039-11044.

69. Delaney, S.P.; Witko, E.M.; Smith, T.M.; Korter, T.M. Investigating tautomeric polymorphism in crystalline anthranilic acid using terahertz spectroscopy and solid-state density functional theory. J. Phys. Chem. A 2012, 116, 8051-8057.

70. Charron, D.M.; Ajito, K.; Kim, J.-Y.; Ueno, Y. Chemical mapping of pharmaceutical cocrystals using terahertz spectroscopic imaging. Anal. Chem. 2013, 85, 1980-1984.

71. Delaney, S.P.; Pan, D.; Yin, S.X.; Smith, T.M.; Korter, T.M. Evaluating the roles of conformational strain and cohesive binding in crystalline polymorphs of aripiprazole. Cryst. Growth Des. 2013, 13, 2943-2952.

72. Takahashi, M.; Ishikawa, Y. Translational vibrations between chains of hydrogen-bonded molecules in solid-state aspirin form I. Chem. Phys. Lett. 2013, 576, 21-25.

73. Nangia, A. Conformational polymorphism in organic crystals. Acc. Chem. Res. 2008, 41, 595-604.

74. Cruz-Cabeza, A.J.; Bernstein, J. Conformational polymorphism. Chem. Rev. 2014, 114, 2170-2191.

75. Braun, D.E.; Gelbrich, T.; Kahlenberg, V.; Laus, G.; Wieser, J.; Griesser, U.J. Packing polymorphism of a conformationally flexible molecule (aprepitant). New J. Chem. 2008, 32, 1677-1685.

76. Brittain, H.G. Polymorphism and solvatomorphism 2007. J. Pharm. Sci. 2009, 98, 1617-1642.

77. Choudhury, A.R.; Islam, K.; Kirchner, M.T.; Mehta, G.; Row, T.N.G. In situ cryocrystallization of diphenyl ether: $\mathrm{C}-\mathrm{H} \cdots \pi$ mediated polymorphic forms. J. Am. Chem. Soc. 2004, 126, 12274-12275.

78. Constable, E.C.; Housecroft, C.E.; Neuburger, M.; Schaffner, S.; Shardlow, E.J. Polymorphs of 4'-(hex-5-ynyloxy)-2,2':6',2"-terpyridine: Structural diversity arising from weak intermolecular interactions in the solid state. CrystEngComm 2005, 7, 599-602.

79. Omondi, B.; Fernandes, M.A.; Layh, M.; Levendis, D.C.; Look, J.L.; Mkwizu, T.S.P. Polymorphism and phase transformations in 2,6-disubstituted N-phenylformamides: The influence of hydrogen bonding, chloro-methyl exchange, intermolecular interactions and disorder. CrystEngComm 2005, 7, 690-700.

80. Chopra, D.; Nagarajan, K.; Row, T.N.G. Polymorphism in 1-(4-fluorophenyl)-3,6,6-trimethyl-2phenyl-1,5,6,7-tetrahydro-4H-indol-4-one: A subtle interplay of weak intermolecular interactions. Cryst. Growth Des. 2005, 5, 1035-1039.

81. Choudhury, A.R.; Nagarajan, K.; Row, T.N.G. Solvent mediated centric/non-centric polymorph pairs of an indole derivative: Subtle variation of $\mathrm{C}-\mathrm{H} \cdots \mathrm{O}$ hydrogen bonds and $\mathrm{C}-\mathrm{H} \cdots \pi$ interactions. CrystEngComm 2006, 8, 482-488.

82. Hulme, A.T.; Johnston, A.; Florence, A.J.; Fernandes, P.; Shankland, K.; Bedford, C.T.; Welch, G.W.A.; Sadiq, G.; Haynes, D.A.; Motherwell, W.D.S.; et al. Search for a predicted hydrogen bonding motif-A multidisciplinary investigation into the polymorphism of 3-azabicyclo[3.3.1]nonane-2,4-dione. J. Am. Chem. Soc. 2007, 129, 3649-3657. 
83. Sardo, M.; Amado, A.M.; Ribeiro-Claro, P.J.A. Hydrogen bonding in nitrofurantoin polymorphs: A computation-assisted spectroscopic study. J. Raman Spectrosc. 2009, 40, 1956-1965.

84. Yu, F.; Yang, G.; Su, Z. The effect of multiple weak interactions on the charge transport ability in polymorphs. Synth. Met. 2011, 161, 1073-1078.

85. Tewari, A.K.; Singh, V.P.; Dubey, R.; Puerta, C.; Valerga, P.; Verma, R. Importance of weak interactions in developing 1,3-bis(4,6-dimethyl-1H-nicotinonitrile-1-yl)1,3-dioxy propane polymorphs. Spectrochim. Acta Part A Mol. Biomol. Spectrosc. 2011, 79, 1267-1275.

86. Flakus, H.T.; Hachła, B. Polarized IR spectra of the hydrogen bond in two different oxindole polymorphs with cyclic dimers in their lattices. J. Phys. Chem. A 2011, 115, 12150-12160.

87. Kumar, S.S.; Nangia, A. A new conformational polymorph of $\mathrm{N}$-acetyl-L-cysteine. The role of

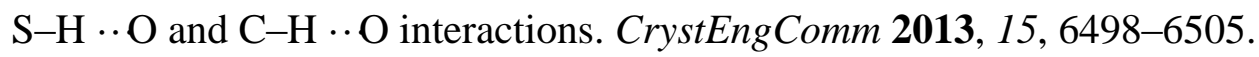

88. Vishweshwar, P.; McMahon, J.A.; Oliveira, M.; Peterson, M.L.; Zaworotko, M.J. The predictably elusive form II of aspirin. J. Am. Chem. Soc. 2005, 127, 16802-16803.

89. Wheatley, P.J. The crystal and molecular structure of aspirin. J. Chem. Soc. 1964, 6036-6048.

90. Cabezas, C.; Alonso, J.L.; López, J.C.; Mata, S. Unveiling the shape of aspirin in the gas phase. Angew. Chem. Int. Ed. 2012, 51, 1375-1378.

91. Ouvrard, C.; Price, S.L. Toward crystal structure prediction for conformationally flexible molecules: The headaches illustrated by aspirin. Cryst. Growth Des. 2004, 4, 1119-1127.

92. Bond, A.D.; Boese, R.; Desiraju, G.R. On the polymorphism of aspirin. Angew. Chem. Int. Ed. 2007, 46, 615-617.

93. Bond, A.D.; Boese, R.; Desiraju, G.R. On the polymorphism of aspirin: Crystalline aspirin as intergrowths of two "polymorphic" domains. Angew. Chem. Int. Ed. 2007, 46, 618-622.

94. Wen, S.; Beran, G.J.O. Accidental degeneracy in crystalline aspirin: New insights from high-level ab initio calculations. Cryst. Growth Des. 2012, 12, 2169-2172.

95. Cui, X.; Rohl, A.L.; Shtukenberg, A.; Kahr, B. Twisted aspirin crystals. J. Am. Chem. Soc. 2013, 135, 3395-3398.

96. Palik, E.D. History of far-infrared research. I. The Rubens era. J. Opt. Soc. Am. 1977, 67, $857-865$.

97. Grinsburg, N. History of far-infrared research. II. The grating era, 1925-1960. J. Opt. Soc. Am. 1977, 67, 865-871.

98. Smith, P.R.; Auston, D.H.; Nuss, M.C. Subpicosecond photoconducting dipole antennas. IEEE J. Quant. Electron. 1988, 24, 255-260.

99. Fattinger, C.; Grischkowsky, D. Terahertz beams. Appl. Phys. Lett. 1989, 54, 490-492.

100. Saykally, R.J.; Blake, G.A. Molecular interactions and hydrogen bond tunneling dynamics: Some new perspectives. Science 1993, 259, 1570-1575.

101. Yu, B.L.; Yang, Y.; Zeng, F.; Xin, X.; Alfano, R.R. Reorientation of the $\mathrm{H}_{2} \mathrm{O}$ cage studied by terahertz time-domain spectroscopy. Appl. Phys. Lett. 2005, 86, doi:10.1063/1.1862788.

102. Walther, M.; Fischer, B.; Schall, M.; Helm, H.; Jepsen, P.U. Far-infrared vibrational spectra of all-trans, 9-cis and 13-cis retinal measured by THz time-domain spectroscopy. Chem. Phys. Lett. 2000, 332, 389-395.

103. Markelz, A.G.; Roitberg, A.; Heilweil, E.J. Pulsed terahertz spectroscopy of DNA, bovine serum albumin and collagen between 0.1 and 2.0 THz. Chem. Phys. Lett. 2000, 320, 42-48. 
104. Möller, K.D.; Rothschild, W.G. Far-Infrared Spectroscopy; Wiley-Interscience: New York, NY, USA, 1971.

105. Frank, W.F.X.; Fiedler, H. On the problem of direct observation of H-bridge interactions in polymers by FIR spectroscopy. Infrared Phys. 1979, 19, 481-489.

106. Shen, D.Y.; Pollack, S.K.; Hsu, S.L. Far-infrared study of hydrogen bonding in semicrystalline polyurethane. Macromolecules 1989, 22, 2564-2569.

107. Kato, T.; Shirota, H. Intermolecular vibrational modes and orientational dynamics of cooperative hydrogen-bonding dimer of 7-azaindole in solution. J. Chem. Phys. 2011, 134, doi:10.1063/1.3583642.

108. Morgan, J.; Warren, B.E. X-ray analysis of the structure of water. J. Chem. Phys. 1938, 6, 666-673.

109. Brady, G.W.; Romanow, W.J. Structure of water. J. Chem. Phys. 1960, 32, doi:10.1063/1.1700931.

110. Cartwright, C.H. Hindered rotation in liquid $\mathrm{H}_{2} \mathrm{O}$ and $\mathrm{D}_{2} \mathrm{O}$. Phys. Rev. 1936, 49, 470-471.

111. Falk, M.; Giguère, P.A. Infrared spectrum of the $\mathrm{H}_{3} \mathrm{O}^{+}$ion in aqueous solutions. Can. J. Chem. 1957, 35, 1195-1204.

112. Walrafen, G.E. Raman spectral studies of water structure. J. Chem. Phys. 1964, 40, 3249-3256.

113. Dunning, T.H., Jr. Gaussian basis sets for use in correlated molecular calculations. I. The atoms boron through neon and hydrogen. J. Chem. Phys. 1989, 90, 1007-1023.

114. Woon, D.E.; Dunning, T.H., Jr. Gaussian basis sets for use in correlated molecular calculations. III. The atoms aluminum through argon. J. Chem. Phys. 1993, 98, 1358-1371.

115. Bondi, A. Van der Waals volumes and radii. J. Phys. Chem. 1964, 68, 441-451.

116. Yokouchi, M.; Chatani, Y.; Tadokoro, H.; Teranishi, K.; Tani, H. Structural studies of polyesters: 5. Molecular and crystal structures of optically active and racemic poly( $\beta$-hydroxybutyrate). Polymer 1973, 14, 267-272.

117. Iwata, T.; Aoyagi, Y.; Tanaka, T.; Fujita, M.; Takeuchi, A.; Suzuki, Y.; Uesugi, K. Microbeam $\mathrm{X}$-ray diffraction and enzymatic degradation of poly[(R)-3-hydroxybutyrate] fibers with two kinds of molecular conformations. Macromolecules 2006, 39, 5789-5795.

118. Yamamoto, S.; Morisawa, Y.; Sato, H.; Hoshina, H.; Ozaki, Y. Quantum mechanical interpretation of intermolecular vibrational modes of crystalline poly- $(R)-3$-hydroxybutyrate observed in low-frequency Raman and terahertz spectra. J. Phys. Chem. B 2013, 117, 2180-2187.

119. Takahashi, M.; Kawazoe, Y.; Ishikawa, Y.; Ito, H. Interpretation of temperature-dependent low frequency vibrational spectrum of solid-state benzoic acid dimer. Chem. Phys. Lett. 2009, 479, 211-217.

120. Fumino, K.; Reichert, E.; Wittler, K.; Hempelmann, R.; Ludwig, R. Low-frequency vibrational modes of protic molten salts and ionic liquids: Detecting and quantifying hydrogen bonds. Angew. Chem. Int. Ed. 2012, 51, 6236-6240.

121. Xu, W.; Angell, C.A. Solvent-free electrolytes with aqueous solution-like conductivities. Science 2003, 302, 422-425.

122. Yoshizawa, M.; Xu, W.; Angell, C.A. Ionic liquids by proton transfer: Vapor pressure, conductivity, and the relevance of $\Delta \mathrm{p} K_{a}$ from aqueous solutions. J. Am. Chem. Soc. 2003, 125, 15411-15419.

123. Angell, C.A.; Byrne, N.; Belieres, J.-P. Parallel developments in aprotic and protic ionic liquids: Physical chemistry and applications. Acc. Chem. Res. 2007, 40, 1228-1236. 
124. Nuthakki, B.; Greaves, T.L.; Krodkiewska, I.; Weerawardena, A.; Burgar, M.I.; Mulder, R.J.; Drummond, C.J. Protic ionic liquids and iconicity. Aust. J. Chem. 2007, 60, 21-28.

125. Greaves, T.L.; Drummond, C.J. Protic ionic liquids: Properties and applications. Chem. Rev. 2008, 108, 206-237.

126. Fumino, K.; Wulf, A.; Ludwig, R. Hydrogen bonding in protic ionic liquids: Reminiscent of water. Angew. Chem. Int. Ed. 2009, 48, 3184-3186.

127. Stoimenovski, J.; Izgorodina, E.I.; MacFarlane, D.R. Ionicity and proton transfer in protic ionic liquids. Phys. Chem. Chem. Phys. 2010, 12, 10341-10347.

128. Bodo, E.; Postorino, P.; Mangialardo, S.; Piacente, G.; Ramondo, F.; Bosi, F.; Ballirano, P.; Caminiti, R. Structure of the molten salt methyl ammonium nitrate explored by experiments and theory. J. Phys. Chem. B 2011, 115, 13149-13161.

129. Krüger, M.; Bründermann, E.; Funkner, S.; Weingärtner, H.; Havenith, M. Communications: Polarity fluctuations of the protic ionic liquid ethylammonium nitrate in the terahertz regime. J. Chem. Phys. 2010, 132, doi:10.1063/1.3352585.

130. Abdul-Sada, A.K.; Greenway, A.M.; Hitchcock, P.B.; Mohammed, T.J.; Seddon, K.R.; Zora, J.A. Upon the structure of room temperature halogenoaluminate ionic liquids. J. Chem. Soc. Chem. Commun. 1986, 1753-1754.

131. Wulf, A.; Fumino, K.; Ludwig, R. Spectroscopic evidence for an enhanced anion-cation interaction from hydrogen bonding in pure imidazolium ionic liquids. Angew. Chem. Int. Ed. 2010, 49, 449-453.

132. Fumino, K.; Wulf, A.; Ludwig, R. The cation-anion interaction in ionic liquids probed by far-infrared spectroscopy. Angew. Chem. Int. Ed. 2008, 47, 3830-3834.

133. Wulf, A.; Fumino, K.; Ludwig, R.; Taday, P.F. Combined THz, FIR and Raman spectroscopy studies of imidazolium-based ionic liquids covering the frequency range $2-300 \mathrm{~cm}^{-1}$. ChemPhysChem 2010, 11, 349-353.

134. Roth, C.; Peppel, T.; Fumino, K.; Köckerling, M.; Ludwig, R. The importance of hydrogen bonds for the structure of ionic liquids: Single-crystal X-ray diffraction and transmission and attenuated total reflection spectroscopy in the terahertz region. Angew. Chem. Int. Ed. 2010, 49, 10221-10224.

135. Zhao, Y.; Truhlar, D.G. How well can new-generation density functional methods describe stacking interactions in biological systems? Phys. Chem. Chem. Phys. 2005, 7, 2701-2705.

136. Hazra, M.K. Inter-molecular vibrational frequencies of doubly hydrogen-bonded complexes involving 2-pyridone: Reliability of few selected theoretical level of calculations. Chem. Phys. Lett. 2009, 473, 10-16.

137. Dkhissi, A.; Blossey, R. Performance of DFT/MPWB1K for stacking and H-bonding interactions. Chem. Phys. Lett. 2007, 439, 35-39.

138. Antony, J.; Helden, G.; Meijer, G.; Schmidt, B. Anharmonic midinfrared vibrational spectra of benzoic acid monomer and dimer. J. Chem. Phys. 2005, 123, doi:10.1063/1.1947191.

139. Takahashi, M.; Ishikawa, Y.; Nishizawa, J.; Ito, H. Low-frequency vibrational modes of riboflavin and related compounds. Chem. Phys. Lett. 2005, 401, 475-482. 
140. Hakey, P.M.; Allis, D.G.; Hudson, M.R.; Ouellette, W.; Korter, T.M. Investigation of $(1 R, 2 S)$-(-)-ephedrine by cryogenic terahertz spectroscopy and solid-state density functional theory. ChemPhysChem 2009, 10, 2434-2444.

141. Mourik, T.; Duijneveldt, F.B. Ab initio calculations on the $\mathrm{C}-\mathrm{H} \cdots \mathrm{O}$ hydrogen-bonded systems $\mathrm{CH}_{4}-\mathrm{H}_{2} \mathrm{O}, \mathrm{CH}_{3} \mathrm{NH}_{2}-\mathrm{H}_{2} \mathrm{O}, \mathrm{CH}_{3} \mathrm{NH}_{3}{ }^{+}-\mathrm{H}_{2} \mathrm{O}$. J. Mol. Struct. THEOCHEM 1995, 341, 63-73.

142. Jurečka, P.; Šponer, J.; Černý, J.; Hobza, P. Benchmark database of accurate (MP2 and CCSD(T) complete basis set limit) interaction energies of small model complexes, DNA base pairs, and amino acid pairs. Phys. Chem. Chem. Phys. 2006, 8, 1985-1993.

143. Cybulski, S.M.; Lytle, M.L. The origin of deficiency of the supermolecule second-order Møller-Plesset approach for evaluating interaction energies. J. Chem. Phys. 2007, 127, doi:10.1063/1.2795693.

144. Zhao, Y.; Schulz, N.E.; Truhlar, D.G. Exchange-correlation functional with broad accuracy for metallic and nonmetallic compounds, kinetics and noncovalent interactions. J. Chem. Phys. 2005, 123, 161103:1-161103:4.

145. Zhao, Y.; Schultz, N.E.; Truhlar, D.G. Design of density functionals by combining the method of constraint satisfaction with parametrization for thermochemistry, thermochemical kinetics, and noncovalent interactions. J. Chem. Theory Comput. 2006, 2, 364-382.

146. Zhao, Y.; Truhlar, D.G. The M06 suite of density functionals for main group thermochemistry, thermochemical kinetics, noncovalent interactions, excited states, and transition elements: Two new functionals and systematic testing of four M06-class functionals and 12 other functionals. Theor. Chem. Acc. 2008, 120, 215-241.

147. Zhao, Y.; Truhlar, D.G. Density functionals with broad applicability in chemistry. Acc. Chem. Res. 2008, 41, 157-167.

148. Takahashi, K. Theoretical study on the effect of intramolecular hydrogen bonding on $\mathrm{OH}$ stretching overtone decay lifetime of ethylene glycol, 1,3-propanediol, and 1,4-butanediol. Phys. Chem. Chem. Phys. 2010, 12, 13950-13961.

149. Mohamed, M.N.A.; Watts, H.D.; Guo, J.; Catchmark, J.M.; Kubicki, J.D. MP2, density functional theory, and molecular mechanical calculations of $\mathrm{C}-\mathrm{H} \cdots \pi$ and hydrogen bond interactions in a cellulose-binding module-cellulose model system. Carbohydrate Res. 2010, 345, 1741-1751.

150. Cockett, M.C.R.; Miyazaki, M.; Tanabe, K.; Fujii, M. Isomer selective IR-UV depletion spectroscopy of 4-fluorotoluene- $\mathrm{NH}_{3}$ : Evidence for $\pi$-proton-acceptor and linear hydrogen-bonded complexes. Phys. Chem. Chem. Phys. 2011, 13, 15633-15638.

151. Schmies, M.; Patzer, A.; Fujii, M.; Dopfer, O. Structures and IR/UV spectra of neutral and ionic phenol- $\operatorname{Ar}_{n}$ cluster isomers $(n \leq 4)$ : Competition between hydrogen bonding and stacking. Phys. Chem. Chem. Phys. 2011, 13, 13926-13941.

152. Kumar, S.; Biswas, P.; Kaul, I.; Das, A. Competition between hydrogen bonding and dispersion interactions in the indole $\cdots$ pyridine dimer and (Indole) $)_{2} \cdots$ pyridine trimer studied in a supersonic jet. J. Phys. Chem. A 2011, 115, 7461-7472.

153. Ivanova, B.B. Solid-state Raman spectra of non-centrosymmetric crystals-theoretical vs. experimental study towards an application in THz-regime. J. Mol. Struct. 2012, 1016, 47-54. 
154. Vianello, R.; Mavri, J. Microsolvation of the histamine monocation in aqueous solution: The effect on structure, hydrogen bonding ability and vibrational spectrum. New J. Chem. 2012, 36, 954-962.

155. Lippert, K.M.; Hof, K.; Gerbig, D.; Ley, D.; Hausmann, H.; Guenther, S.; Schreiner, P.R. Hydrogen-bonding thiourea organocatalysts: The privileged 3,5-bis(trifluoromethy)phenyl group. Eur. J. Org. Chem. 2012, 2012, 5919-5927.

156. León, I.; Cocinero, E.J.; Rijs, A.M.; Millán, J.; Alonso, E.; Lesarri, A.; Fernández, J.A. Formation of water polyhedrons in propofol-water clusters. Phys. Chem. Chem. Phys. 2013, 15, $568-575$.

157. Blaser, S.; Ottiger, P.; Frey, H.-M.; Leutwyler, $\mathrm{S} . \mathrm{NH}_{3}$ as a strong H-bond donor in singly- and doubly-bridged ammonia solvent clusters: 2-Pyridone $\left(\mathrm{NH}_{3}\right)_{n}, n=1-3$. J. Phys. Chem. A 2013, 117, 7523-7534.

158. Jaeqx, S.; Du, W.; Meijer, E.J.; Oomens, J.; Rijs, A.M. Conformational study of Z-Glu-OH and Z-Arg-OH: Dispersion interactions versus conventional hydrogen bonding. J. Phys. Chem. A 2013, 117, 1216-1227.

159. León, I.; Millán, J.; Cocinero, E.J.; Lesarri, A.; Fernández, J.A. Transition from planar to nonplanar hydrogen bond networks in the solvation of aromatic dimers: $\operatorname{Propofol}_{2}-\left(\mathrm{H}_{2} \mathrm{O}\right)_{2-4} . J$. Phys. Chem. A 2013, 117, 3396-3404.

160. Kohn, W.; Sham, L.J. Self-consistent equations including exchange and correlation effects. Phys. Rev. A 1965, 140, 1133-1138.

161. Parr, R.G.; Yang, W. Density-Functional Theory of Atoms and Molecules; Oxford University Press: New York, NY, USA, 1989.

162. Koch, W.; Holthausen, M.C. A Chemist's Guide to Density Functional Theory, 2nd ed.; Wiley-VCH: Weinheim, Germany, 2001.

163. Grimme, S. Semiempirical GGA-type density functional constructed with a long-range dispersion correction. J. Comput. Chem. 2006, 27, 1787-1799.

164. Grimme, S.; Antony, J.; Ehrlich, S.; Krieg, H. A consistent and accurate ab initio parametrization of density functional dispersion correction (DFT-D) for the 94 elements H-Pu. J. Chem. Phys. 2010, 132, doi:10.1063/1.3382344.

165. Tkatchenko, A.; Scheffler, M. Accurate molecular van der Waals interactions from ground-state electron density and free-atom reference data. Phys. Rev. Lett. 2009, 102, doi:10.1103/ PhysRevLett.102.073005.

166. Lilienfeld, O.A.; Tkatchenko, A. Two- and three-body interatomic dispersion energy contributions to binding in molecules and solids. J. Chem. Phys. 2010, 132, doi:10.1063/1.3432765.

167. Al-Saidi, W.A.; Voora, V.K.; Jordan, K.D. An assessment of the vdW-TS method for extended systems. J. Chem. Theory Comput. 2012, 8, 1503-1513.

168. Hujo, W.; Grimme, S. Comparison of the performance of dispersion-corrected density functional theory for weak hydrogen bonds. Phys. Chem. Chem. Phys. 2011, 13, 13942-13950.

169. Civalleri, B.; Zicovich-Wilson, C.M.; Valenzano, L.; Ugliengo, P. B3LYP augmented with an empirical dispersion term (B3LYP-D*) as applied to molecular crystals. CrystEngComm 2008, 10, 1693-1694. 
170. King, M.D.; Buchanan, W.D.; Korter, T.M. Application of London-type dispersion corrections to the solid-state density functional theory simulation of the terahertz spectra of crystalline pharmaceuticals. Phys. Chem. Chem. Phys. 2011, 13, 4250-4259.

171. King, M.D.; Korter, T.M. Application of London-type dispersion corrections in solid-state density functional theory for predicting the temperature-dependence of crystal structures and terahertz spectra. Cryst. Growth Des. 2011, 11, 2006-2010.

172. King, M.D.; Ouellette, W.; Korter, T.M. Noncovalent interactions in paired DNA nucleobases investigated by terahertz spectroscopy and solid-state density functional theory. J. Phys. Chem. A 2011, 115, 9467-9478.

173. Takahashi, M.; Ishikawa, Y.; Ito, H. The dispersion correction and weak-hydrogen-bond network in low-frequency vibration of solid-state salicylic acid. Chem. Phys. Lett. 2012, 531, 98-104.

174. Beyer, T.; Price, S.L. The errors in lattice energy minimisation studies: Sensitivity to experimental variations in the molecular structure of paracetamol. CrystEngComm 2000, 34, 1-8.

175. Pulay, P. Ab initio calculation of force constants and equilibrium geometries in polyatomic molecules. I. Theory. Mol. Phys. 1969, 17, 197-204.

176. Hagen, K.; Cross, V.R.; Hedberg, K. The molecular structure of selenonyl fluoride, $\mathrm{SeO}_{2} \mathrm{~F}_{2}$, and sulfuryl fluoride, $\mathrm{SO}_{2} \mathrm{~F}_{2}$, as determined by gas-phase electron diffraction. J. Mol. Struct. 1978, 44, 187-193.

177. Yamaguchi, Y.; Schaefer, H.F., III. A systematic theoretical study of harmonic vibrational frequencies: The ammonium ion $\mathrm{NH}_{4}{ }^{+}$and other simple molecules. J. Chem. Phys. 1980, 73, 2310-2318.

178. Yamaguchi, Y.; Frisch, M.; Gaw, J.; Schaefer, H.F., III.; Binkley, J.S. Analytic evaluation and basis set dependence of intensities of infrared spectra. J. Chem. Phys. 1986, 84, 2262-2278.

179. Hehre, W.J.; Radom, L.; Schleyer, P.R.; Pople, J. Ab Initio Molecular Orbital Theory, 1st ed.; Wiley-Interscience: New York, NY, USA, 1986.

180. King, M.D.; Buchanan, W.D.; Korter, T.M. Investigating the anharmonicity of lattice vibrations in water-containing molecular crystals through the terahertz spectroscopy of L-serine monohydrate. J. Phys. Chem. A 2010, 114, 9570-9578.

181. Brittain, H.G. Polymorphism in Pharmaceutical Solids; Brittain, H.G., Ed.; Marcel Dekker: New York, NY, USA, 1999.

182. Kowal, A.T. First-principles computation of the anharmonic vibrational spectra of sulfuryl halides $\mathrm{SO}_{2} \mathrm{X}_{2}$ (X = F, Cl, Br). Chem. Phys. 2006, 324, 359-366.

183. Chaban, G.M.; Jung, J.O.; Gerber, R.B. Anharmonic vibrational spectroscopy of hydrogen-bonded systems directly computed from ab initio potential surfaces: $\left(\mathrm{H}_{2} \mathrm{O}\right)_{n}, n=2,3 ; \mathrm{Cl}^{-}\left(\mathrm{H}_{2} \mathrm{O}\right)_{n}, n=1,2$; $\mathrm{H}^{+}\left(\mathrm{H}_{2} \mathrm{O}\right)_{n}, n=1,2 ; \mathrm{H}_{2} \mathrm{O}-\mathrm{CH}_{3} \mathrm{OH}$. J. Phys. Chem. A 2000, 104, 2772-2779.

184. Petković, M. Infrared spectroscopy of $\mathrm{ClONO}_{2}$ and $\mathrm{BrONO}_{2}$ investigated by means of anharmonic force fields. Chem. Phys. 2007, 331, 438-446.

185. Garrett, R.H.; Grisham, C.M. Biochemistry, 2nd ed.; Sanders College Publishing: Fort Worth, TX, USA, 1999.

186. Shen, Y.C.; Upadhya, P.C.; Linfield, E.H.; Davies, A.G. Temperature-dependent low-frequency vibrational spectra of purine and adenine. Appl. Phys. Lett. 2003, 82, 2350-2352. 
187. Plazanet, M.; Fukushima, N.; Johnson, M.R. Modelling molecular vibrations in extended hydrogen-bonded networks - Crystalline bases of RNA and DNA and the nucleosides. Chem. Phys. 2002, 280, 53-70.

188. Giese, B.; McNaughton, D. Surface-enhanced Raman spectroscopic and density functional theory study of adenine adsorption to silver surfaces. J. Phys. Chem. B 2002, 106, 101-112.

189. Nowak, M.J.; Lapinski, L.; Kwiatkowski, J.S.; Leszczyński, J. Molecular structure and infrared spectra of adenine. Experimental matrix isolation and density functional theory study of adenine ${ }^{15} \mathrm{~N}$ isotopomers. J. Phys. Chem. 1996, 100, 3527-3534.

190. Gaigeot, M.-P.; Leulliot, N.; Ghomi, M.; Jobic, H.; Coulombeau, C.; Bouloussa, O. Analysis of the structural and vibrational properties of RNA building blocks by means of neutron inelastic scattering and density functional theory calculations. Chem. Phys. 2000, 261, 217-237.

191. Hielscher, R.; Hellwig, P. The temperature-dependent hydrogen-bonding signature of lipids monitored in the far-infrared domain. ChemPhysChem 2010, 11, 435-441.

192. Takahashi, M.; Kawazoe, Y.; Ishikawa, Y.; Ito, H. Low-frequency vibrations of crystalline a, $\alpha$-trehalose dihydrate. Chem. Phys. Lett. 2006, 429, 371-377.

193. Rungsawang, R.; Ueno, Y.; Tomita, I.; Ajito, K. Angle-dependent terahertz time-domain spectroscopy of amino acid single crystals. J. Phys. Chem. B 2006, 110, 21259-21263.

194. Whitley, V.H.; Hooks, D.E.; Ramos, K.J.; O’Hara, J.F.; Azad, A.K.; Taylor, A.J.; Barber, J.; Averitt, R.D. Polarization orientation dependence of the far infrared spectra of oriented single crystals of 1,3,5-trinitro-S-triazine (RDX) using terahertz time-domain spectroscopy. Anal. Bioanal. Chem. 2009, 395, 315-322.

195. Hoshina, H.; Morisawa, Y.; Sato, H.; Minamide, H.; Noda, I.; Ozaki, Y.; Otani, C. Polarization and temperature dependent spectra of poly(3-hydroxyalkanoate)s measured at terahertz frequencies. Phys. Chem. Chem. Phys. 2011, 13, 9173-9179.

196. Melinger, J.S.; Laman, N.; Harsha, S.S.; Cheng, S.; Grischkowsky, D. High-resolution waveguide terahertz spectroscopy of partially oriented organic polycrystalline films. J. Phys. Chem. A 2007, 111, 10977-10987.

197. Laman, N.; Harsha, S.S.; Grischkowsky, D. Narrow-line waveguide terahertz time-domain spectroscopy of aspirin and aspirin precursors. Appl. Spectrosc. 2008, 62, 319-326.

198. Melinger, J.S.; Laman, N.; Grischkowsky, D. The underlying terahertz vibrational spectrum of explosive solids. Appl. Phys. Lett. 2008, 93, doi:10.1063/1.2949068.

(C) 2014 by the authors; licensee MDPI, Basel, Switzerland. This article is an open access article distributed under the terms and conditions of the Creative Commons Attribution license (http://creativecommons.org/licenses/by/3.0/). 